\title{
An Energy-Equivalent Simplified Model for Lateral Nonlinear Vibrations of Coupled Water Turbine Generator Set Shaft-Foundation System
}

\author{
Wanquan Sun ( $\nabla$ sunwanquan75@163.com ) \\ North China Electric Power University \\ Zhiqiang Guo \\ North China Electric Power University \\ Zhenyue Ma \\ Dalian University of Technology
}

\section{Research Article}

Keywords: Coupled water turbine generator set shaft-foundation system (CSFS), Energy equivalent model, Lumped-parameter models, Nonlinear vibration, Model simplification

Posted Date: March 16th, 2021

DOI: https://doi.org/10.21203/rs.3.rs-274422/v1

License: (c) (i) This work is licensed under a Creative Commons Attribution 4.0 International License. Read Full License 


\title{
An energy-equivalent simplified model for lateral nonlinear vibrations of coupled water turbine generator set shaft-foundation system
}

\author{
Wanquan Sun ${ }^{\mathrm{a}, *}$, Zhiqiang Guo ${ }^{\mathrm{a}}$, Zhenyue $\mathrm{Ma}^{\mathrm{b}}$ \\ ( ${ }^{a}$ School of Water Resources and Hydropower Engineering, North China \\ Electric Power University, Beijing 102206, China; \\ ${ }^{b}$ School of Hydraulic Engineering, Faculty of Infrastructure Engineering, Dalian \\ University of Technology, Dalian, Liaoning Province 116024, China) \\ * Corresponding author: Wanquan Sun (Email: sunwanquan75@163.com)
}

\section{Declarations of interest: none}

\begin{abstract}
The traditional whole finite element method (WFEM ${ }^{1}$ ) has several shortcomings, including that it has too many degrees of freedom so the execution is not efficient and is difficult to solve in nonlinear dynamic analysis. In this paper, a novel simplified modeling approach is proposed to investigate the lateral nonlinear vibration characteristics of coupled water turbine generator set shaft-foundation system $\left(\mathrm{CSFS}^{2}\right)$. The simplified coupled model is generated in two stages. First, a more reasonable simplified model for foundation subsystem considering coupling with each other is constructed to simulate the lateral vibrations of guide bearing foundations in hydropower house. Based on the response spectra of WFEM of hydropower house and a constructed energy error objective function, the optimal equivalent parameters of the simplified foundation model are then determined by using the genetic algorithm. Second, considering actions of various nonlinear factors and the pulsating water pressure acting on turbine runner, a nonlinear dynamic differential equations of CSFS based on Lagrange equation are derived. The nonlinear dynamic responses of CSFS using the optimal equivalent model are also compared with the field test data. It is demonstrated that the method proposed to develop the equivalent model is more efficient and more convenient in capturing the nonlinear dynamic behavior of CSFS. In addition, this energy-equivalent model is more adaptable to the stochastic uncertainty and frequency band variation in hydropower station system. Some novel dynamic laws and inner mechanism of the coupled system are also revealed further based on the proposed model.
\end{abstract}

Key words: Coupled water turbine generator set shaft-foundation system (CSFS); Energy equivalent model; Lumped-parameter models; Nonlinear vibration; Model simplification;

\section{Introduction}

The vibration problem of the hydro generator units and its supporting

\footnotetext{
1 WFEM: whole finite element method

${ }^{2}$ CSFS: coupled water turbine generator set shaft-foundation system
} 
structure (i.e. hydropower house foundation) is one of the key issues influencing operational security and stability of hydropower station. Due to the nonlinear dynamic behavior of the hydro generator units and higher complexity of the hydropower house structure, the dynamic analyses for unit system have not long taken account of the vibrational effect of hydropower house. In the past, the vibrations of the hydro generator shaft system and hydropower house have been extensively studied from different perspectives, and the relevant achievements have been quite fruitful [1-9]. In fact, the water turbine generator set and its supporting foundation should be a whole coupling system, which includes not only the shaft-foundation coupling but also the coupling between foundations (as shown in Fig. 1(b)). The oscillation phenomenon of CSFS is also of time-varying and strongly nonlinear stochastic dynamics simultaneously. The strongly nonlinear stochastic vibrations usually come from the direct rotor-bearing-seal interactions [10], random external loads and the nonlinear vibrations of damaged concrete structures, etc. [11]. The effect of foundation vibration is very sensitive to the whole dynamic characteristics of CSFS, a slight perturbation of the system parameters may also cause unpredictable and drastic changes of the coupling nonlinear dynamic behaviors [12]. Accordingly, increased attention has been aroused to perform the coupling dynamic analyses for CSFS in recent years. Wu and Zhang et al. [13, 14] developed a whole coupling model for hydropower station considering hydro-mechanical-electric and structure system, and the corresponding dynamic properties under different operation cases were studied. Zhang and Wang et al. [15] built whole fluid-machinery-concrete structure coupling finite element model and studied the vibration transmission path in the hydropower plant using the time-delayed transfer entropy method. Song and $\mathrm{Ma}$ et al. [16] established the coupling mechanical model including a water turbine generator set and hydropower plant, its transitional running condition is analyzed so as to evaluate the effect of hydropower house foundation and each pad's nonlinear dynamic coefficients on system response. However, it should be noted that the WFEM (just as shown in Fig. 1(a)) has been basically adopted in the coupling system modeling in previous researches. The method of WFEM has shown some disadvantages for the nonlinear dynamic analysis of CSFS: consisting of a high number of elements and degrees-of-freedom, requiring more solving time and greater computational source, this may lead to a difficult solution particularly in some problems with a large number of very small time steps requested (for example, high-frequency time-history dynamic analysis) or sensitivity analysis of parameters variation, etc.

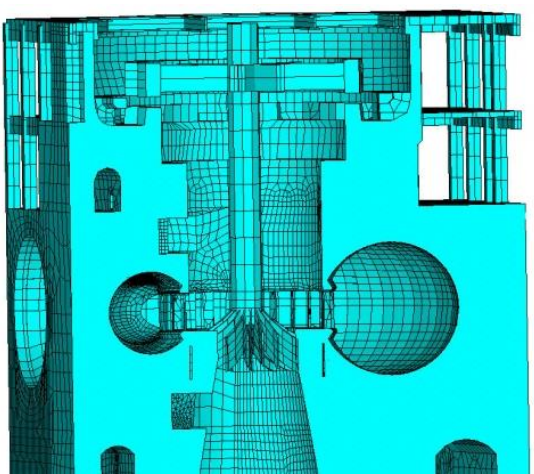

(a)

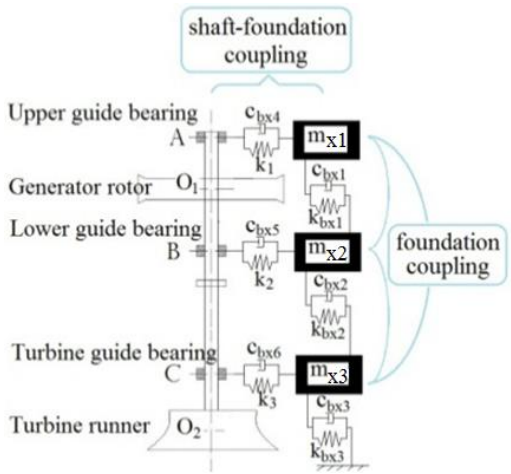

(b)

Fig. 1 (a) Whole finite element model and (b) equivalent simplified model of a coupled water turbine generator set shaft-foundation system

In addition to the above-mentioned WFEM, the most commonly-used 
methods for dynamic structural analysis are the substructure method and lumped parameter method. In contrast, the substructure method and lumped parameter method feature in reducing the system to a smaller set of degrees of freedom, which reduce the computer time and allow the solution of very large problems with limited computer resources. However, since these two methods are specific frequency-dependent (mostly low-order main frequency), they cannot be used directly in broadband vibration analysis or time-domain structural analysis [17], and they are especially difficult for the modeling of CSFS due to the structural complexity and wide response spectrum characteristic. For these reasons too, there has been very little modeling and research on nonlinear vibrations for CSFS in current literatures. So far, only a few linear elastic modal analyses for CSFS have been conducted by using roughly estimated three independent foundation mass models, and not considering the different guide bearing foundations coupling. Hence, for computational efficiency, it is necessary to develop a more reasonable modeling methodology so as to effectively simulate the nonlinear dynamic characteristics for the CSFS.

Actually, in many cases, a detailed and highly accurate model is unnecessary because approximations can be made, while still capturing the mechanisms that operate in the particular case. In this paper, on the basis of the nonlinear stochastic dynamics theory, the study mainly presents a new equivalent simplified model to simulate the CSFS undergoing coupled horizontal motion. The present model could not only capture the nonlinear dynamic behaviors of the coupled shaftfoundation system but also reflect the coupled vibration features of different supporting foundations. This study also supplements the existing dynamic theories and methods for analyzing the CSFS.

The rest of the paper is organized as follows. A more reasonable energyequivalent lumped-parameter model of the foundation subsystem is presented, along with the modeling methodology and principles, in Section 2. Moreover, this section also compares the dynamic responses of a foundation subsystem using the new energy-equivalent model with those obtained by the whole finite element model, by the modal behavior based simplified model. In section 3, based on the novel simplified model of foundation subsystem and considering actions of various nonlinear factors of unit subsystem, the nonlinear coupled dynamic model of the CSFS is established by Lagrange approach. The next section 4 provides a validation of the derived dynamic coupling equations and its numerical solutions of CSFS utilizing field measurement results in previous literature. The energyequivalent simplified model solved numerically has proven to be effective to calculate the coupling vibration and interaction between the unit subsystem and foundation subsystem, and be useful for evaluating the vibration performance of hydropower station subjected to different output conditions. The differences of nonlinear vibration responses between individual shaft system (no coupling foundation) and CSFS models are discussed in Section 5, consisting of amplitude, frequency and stability. The conclusions of this paper are exhibited in section 6 . 


\section{Energy-equivalent model of foundation subsystem}

\subsection{Modeling method for foundation subsystem}

While the WFEM is the most accurate modeling for the supporting foundation of hydropower unit, the supporting foundation system can be simplified if there is no need for a detailed analysis of the hydropower house structure. However, it must be emphasized that the strong coupling effects between different guide bearing foundations should be taken into account in the simplified model, because the vibrations of different foundations will be transmitted and influenced each other through the integral concrete structure of hydropower house. To this end, this study develops a three-layer shear spring-damper-mass model (shown in Fig. 1(b) and called the coupled lumped parameter model) that is used to simplify the vibration behavior and the coupling processes of three guide bearing foundations. The simplified model includes the key nine parameters of equivalent shear stiffness $\left(k_{\mathrm{b} \times 1} \sim k_{\mathrm{b} \times 3}\right)$, equivalent mass $(m \mathrm{~b} 1 \sim m \mathrm{~b} 3)$, and equivalent damping ( $c \mathrm{bx} 1 \sim$ $(\mathrm{b} \times 3)$. An equivalent model is then determined from these optimal equivalent parameters through minimizing an energy error function.

Suppose the foundation subsystem is a linear elastic stochastic system. The proposed method for establishing an optimal energy-equivalent model follows these subsequent steps:

(1) Determination of initial values and optimization ranges for these equivalent parameters. To optimize the equivalent model, the initial parameter values of the equivalent model are established by considering three equivalent criteria. These criteria establish the equivalence between a guide bearing foundation system in WFEM and the simplified foundation model. The optimization range of these equivalent parameters can be specified in a given percentage range of the initial value.

The first criterion is equivalent static displacement to create the initial values of equivalence stiffness ( $\left.k_{\mathrm{b} \times 1} \sim k_{\mathrm{b} \times 3}\right)$, namely the same lateral static load is applied to the foundations of WFEM and simplified model, respectively, so that the lateral displacements of the simplified foundation model are the same as those of WFEM. This can be accomplished by adjusting shear stiffness parameters of the simplified model. Then initial values of the equivalent shear stiffness $\left(k_{\mathrm{bx} 1} \sim k_{\mathrm{bx} 3}\right)$ can be obtained.

The second criterion is equivalent natural frequency to create the initial values of the equivalence mass parameters $\left(m_{\mathrm{b} 1} \sim m_{\mathrm{b} 3}\right)$. Based on the natural frequencies of WFEM and the identified initial values of equivalence stiffness above, the initial values of the equivalence mass parameters for the simplified model can be calculated directly.

The third criterion is equivalent dissipated energy to create the initial values of the equivalence damping $\left(c_{\mathrm{b} \times 1} \sim c_{\mathrm{b} \times 3}\right)$. Some researchers have identified damping ratios of hydropower house through field tests $[8,9,18]$. The range of identified damping ratio is approximately $2 \%$ to $4 \%$. The damping ratio of $3 \%$ is assigned to calculate the initial values of equivalent shear damping coefficients herein. In this case, the values of $c_{\mathrm{bx} 1}, c_{\mathrm{b} \times 2}$ and $c_{\mathrm{b} \times 3}$ are assumed same.

(2) Calculation of response spectrum. By applying a series of harmonic forces corresponding to $\mathrm{N}$ discrete frequencies, the corresponding response spectrums 
can be obtained for the simplified foundation model and WFEM, respectively.

(3) Determine the optimal model. An optimal energy-equivalent foundation model is then determined from optimizing the equivalent parameters to obtain the response spectrum curves closest to those of WFEM, namely the optimal model has the minimum error $\left(\varepsilon_{r}\right)$ of response spectrum curves. The optimization method selected is genetic algorithm herein. The energy error function $\varepsilon_{\mathrm{r}}$ is defined as:

$$
\varepsilon_{r}=\sum_{i=1}^{9} \quad \sum_{j=1}^{N} \sqrt{\left[\left(\frac{M_{e}(j)-M_{a}(j)}{M_{a}(j)}\right)^{2} \times w_{j}\right]_{i}}
$$

where $j$ is serial number of harmonic forces, $M_{e}(j)$ is the response spectrum value of the equivalent model, $M_{a}(j)$ is the response spectrum value of the WFEM, $w_{j}$ is weight, $i$ is serial number of response spectrum (total number is 9) corresponding to each foundation when applying the harmonic forces to the three foundation, respectively.

The sequence of steps in establishing the optimal energy-equivalent model for foundation subsystem is shown graphically in Fig. 2.

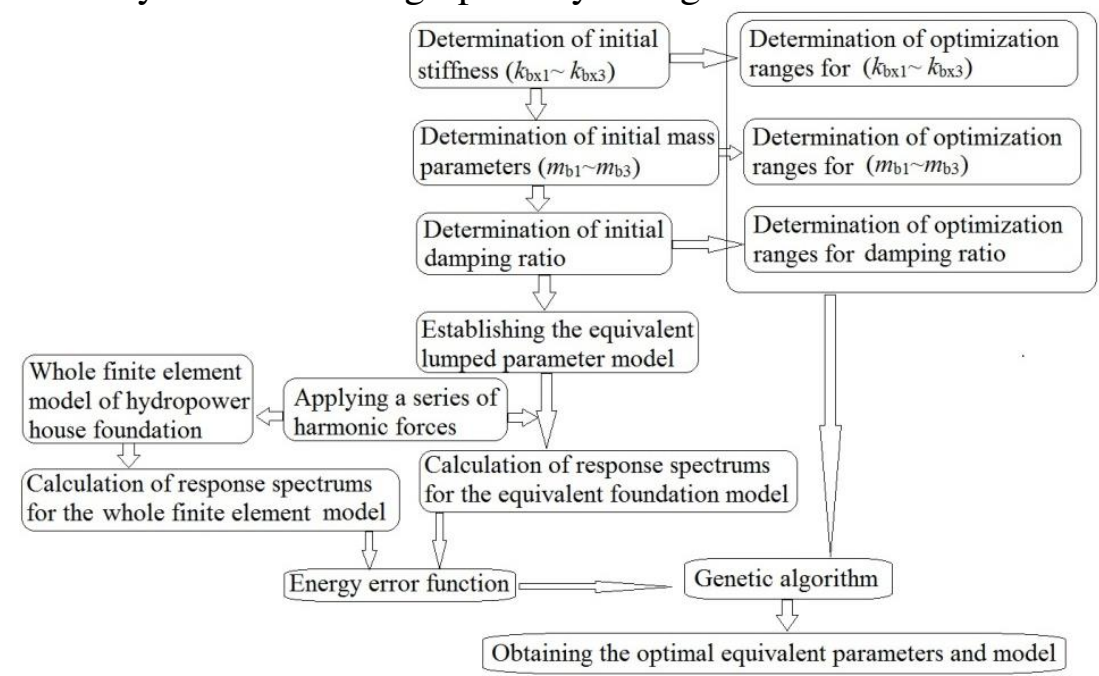

Fig. 2 Procedure for establishing an optimal energy-equivalent model for foundation subsystem

\subsection{Analysis and validation in frequency-domain}

Based on the proposed modeling method, a series of harmonic excitations with same amplitude of $3000 \mathrm{kN}$ and frequencies from $2 \mathrm{~Hz}$ to $60 \mathrm{~Hz}$ are applied to the simplified model and WFEM, respectively. Then the optimal equivalent foundation model is found with genetic algorithm, and its corresponding parameters are obtained and listed in Table 1.

Table 1 Corresponding parameters of the optimal equivalent foundation model.

\begin{tabular}{|c|c|c|c|c|c|}
\hline \multirow{2}{*}{$\mathrm{x}$-direction } & $\begin{array}{r}k \mathrm{bxx}(\mathrm{kN} / \mathrm{m}) \\
1.817 \times 10^{7}\end{array}$ & $\begin{array}{r}k \mathrm{bx} 2(\mathrm{kN} / \mathrm{m}) \\
5.585 \times 10^{7}\end{array}$ & $\begin{array}{c}k \mathrm{bx} 3(\mathrm{kN} / \mathrm{m}) \\
5.11 \times 10^{7}\end{array}$ & $\begin{array}{l}m_{\mathrm{x} 1}(\mathrm{~kg}) \\
5.91 \times 10^{5}\end{array}$ & $\begin{array}{c}m_{\times 2}(\mathrm{~kg}) \\
1.133 \times 10^{7}\end{array}$ \\
\hline & $\begin{array}{c}m_{\mathrm{x} 3}(\mathrm{~kg}) \\
1.265 \times 10^{6}\end{array}$ & $\begin{array}{c}c_{\mathrm{bxl}}(\mathrm{kN} \cdot \mathrm{s} / \mathrm{m}) \\
6.217 \times 10^{3}\end{array}$ & $\begin{array}{c}c_{\mathrm{bx} 2}(\mathrm{kN} \cdot \mathrm{s} / \mathrm{m}) \\
4.773 \times 10^{4}\end{array}$ & $\begin{array}{c}c_{\mathrm{bx} 3}(\mathrm{kN} \cdot \mathrm{s} / \mathrm{m}) \\
1.526 \times 10^{4}\end{array}$ & \\
\hline \multirow{2}{*}{$y$-direction } & $\begin{array}{c}k_{\text {byl }}(\mathrm{kN} / \mathrm{m}) \\
1.62 \times 10^{7}\end{array}$ & $\begin{array}{c}k_{\text {by } 2}(\mathrm{kN} / \mathrm{m}) \\
4.38 \times 10^{7}\end{array}$ & $\begin{array}{c}k_{\text {by3 }}(\mathrm{kN} / \mathrm{m}) \\
5.68 \times 10^{7}\end{array}$ & $\begin{array}{c}m_{\mathrm{y} 1}(\mathrm{~kg}) \\
5.03 \times 10^{5}\end{array}$ & $\begin{array}{l}m_{\mathrm{y} 2}(\mathrm{~kg}) \\
1.05 \times 10^{7}\end{array}$ \\
\hline & $\begin{array}{l}m_{\mathrm{y} 3}(\mathrm{~kg}) \\
1.18 \times 10^{6}\end{array}$ & $\begin{array}{c}\text { byl }(\mathrm{kN} \cdot \mathrm{s} / \mathrm{m}) \\
5.416 \times 10^{3}\end{array}$ & $\begin{array}{c}\text { by } 2(\mathrm{kN} \cdot \mathrm{s} / \mathrm{m}) \\
4.069 \times 10^{4}\end{array}$ & $\begin{array}{c}\text { by3 }(\mathrm{kN} \cdot \mathrm{s} / \mathrm{m}) \\
1.553 \times 10^{4}\end{array}$ & \\
\hline
\end{tabular}

To validate the reasonability of the established energy-equivalent foundation model, another simplified model determined by the conventional modal behavior based method (i.e., the equivalent stiffness and mass parameters are obtained by 
inverse calculation using the few low-order natural vibration frequencies and modes.) is also calculated and compared. Comparisons of the simulated results for the three different modeling methods are performed in frequency-domain and shown in Fig. 3. Note that the results obtained by the optimal energy-equivalent model generally consist well with the WFEM solutions except the very individual result just as Fig. 3(i). While the accuracy of the energy-equivalent model is a little less than that of the modal behavior based simplified model in low frequency $(8 \mathrm{~Hz})$, the energy-equivalent model has the obvious advantage of greater accuracy than the modal behavior based simplified model in medium- and higherfrequency. In some calculations, especially between $16 \mathrm{~Hz}$ and $28 \mathrm{~Hz}$, the results of the modal behavior based model are completely wrong when comparing results of the WFEM, but the results of the energy-equivalent model still keep the higher accuracy.

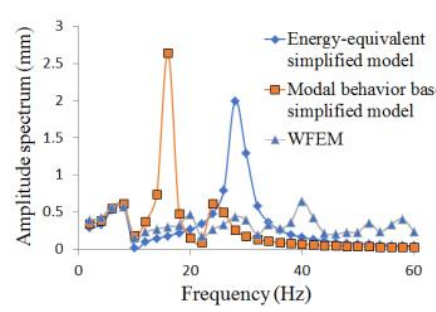

(a) $1-1$

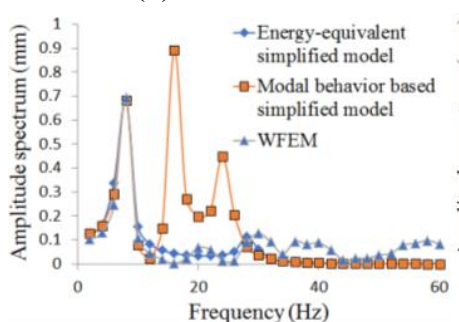

(d) 2-1

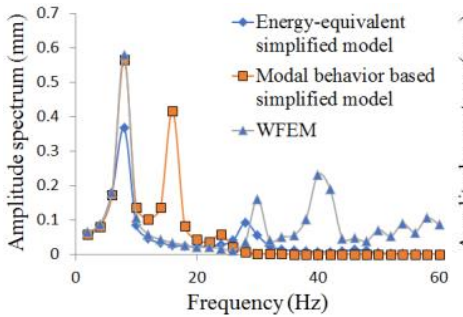

(g) 3-1

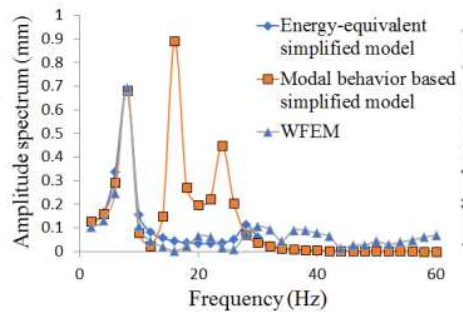

(b) $1-2$

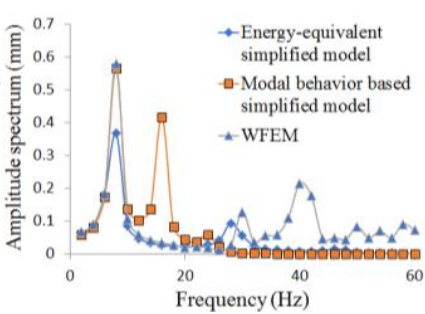

(c) 1-3

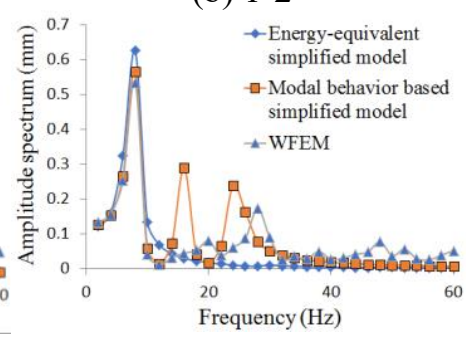

(e) 2-2

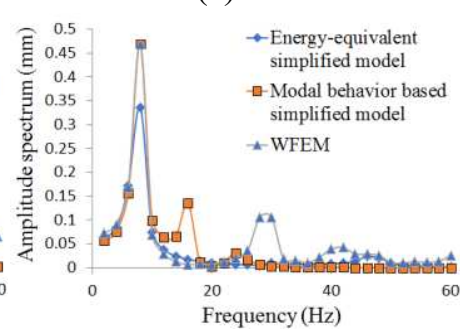

(h) $3-2$

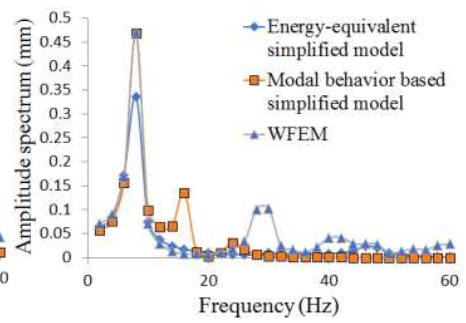

(f) $2-3$

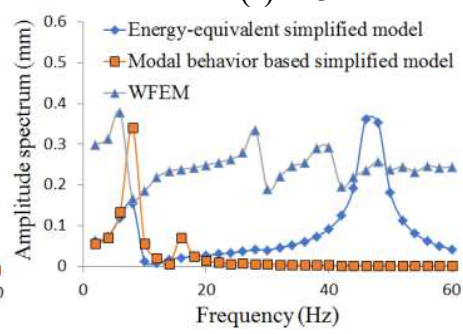

(i) 3-3

Fig. 3 Model comparison for foundation subsystem in frequency-domain (In the subtitle "1, 2, 3 " denote upper guide bearing foundation, lower guide bearing foundation and turbine guide bearing foundation, respectively. The symbol "1-2" means "the dynamic responses of the lower guide bearing foundation when upper guide bearing foundation is subjected to harmonic loadings.")

\subsection{Analysis and validation in time-domain}

In order to further evaluate the established energy-equivalent model, the vibration signals of the foundation subsystem collected from the simulated results of the three different modeling methods are also analyzed and compared in the form of time-domain distribution. For the purpose, two kinds of different random excitations are constructed, including the white noise excitation (i.e., uniform random excitation as shown in Fig. 4) and random excitation with narrow spectral width (as shown in Fig. 5). The constructed random excitations are applied on different foundations respectively, and the acceleration root mean square values 
(Effective values) of the time-history responses of each foundation are then obtained, the results are represented in Fig. 6. As can be seen from Fig. 6, the calculated results of the energy-equivalent simplified model give better approximations to the results of the WFEM than those of the conventional modal behavior based simplified model, whether the values or distribution of the dynamic responses.

In conclusion, the optimal energy-equivalent model could provide an effective method for the more realistic prediction of the dynamic performance of the foundation subsystem and for the dynamic evaluations of the vibration transmission between different guide bearing foundations.

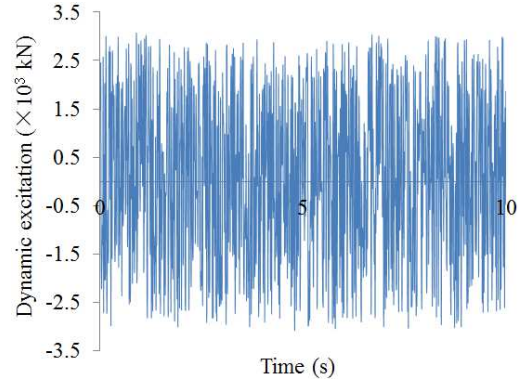

(a)

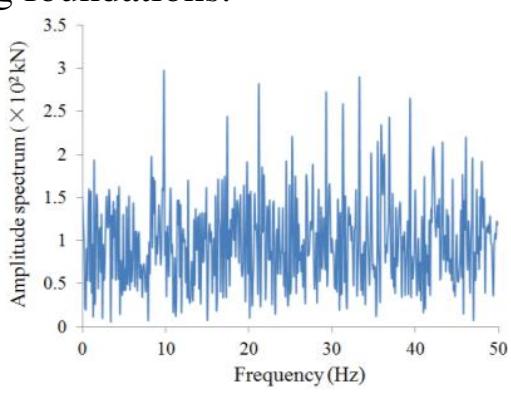

(b)

Fig. 4 The first time-varying excitation constructed by white noise. (a) time-history curve of this excitation; (b) spectrum characteristic of this excitation
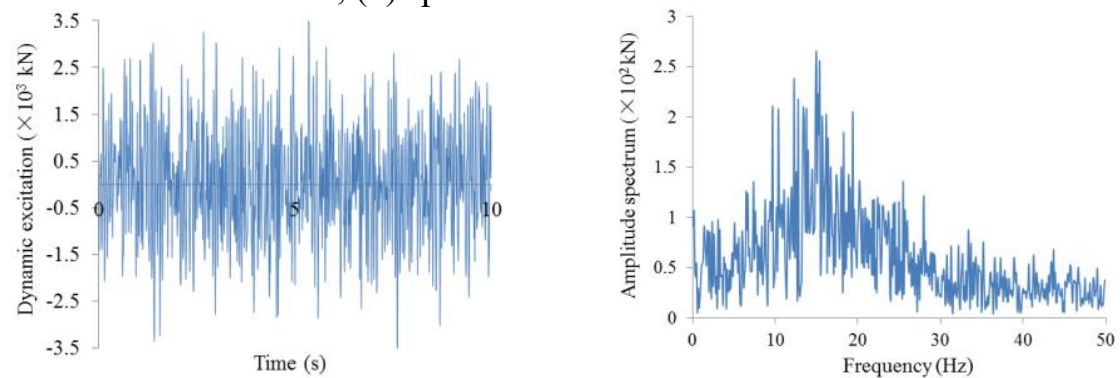

Fig. 5 The second time-varying excitation constructed by bounded noise. (a) time-history curve of this excitation; (b) spectrum characteristic of this excitation
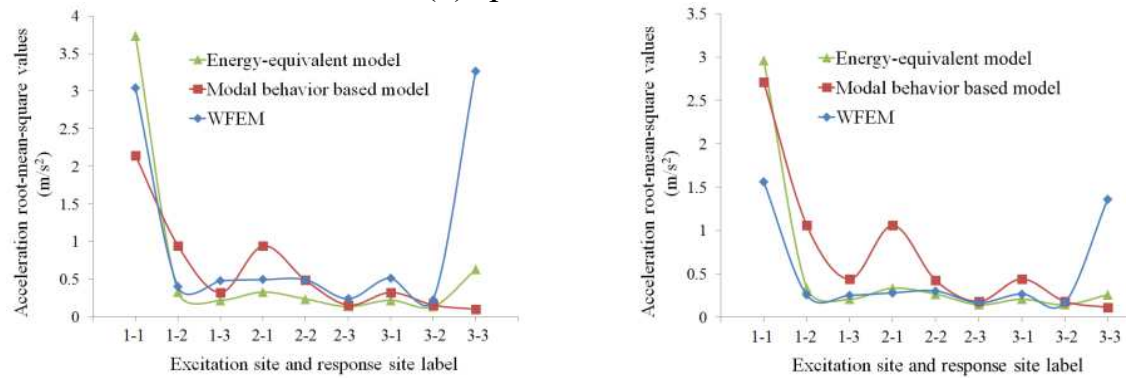

(a) Response to the first random excitation; (b) Response to the second random excitation Fig. 6 Acceleration root-mean-square values of time-history responses for different models (See Fig. 3 for description of the $\mathrm{X}$-axis markers)

\section{Modeling of the whole nonlinear coupled shaft- foundation system}

Based on the established equivalent foundation model, then the foundation subsystem and the nonlinear shaft subsystem may be incorporated into a comprehensive model that can yield coupled dynamics information directly. The nonlinear dynamic differential equations for the CSFS are derived from Lagrange equation, considering the nonlinear effects of rotor unbalance, runner sealing 
force and nonlinear oil film forces, etc. In this study, the lateral vibration of the hydraulic turbine generator set is only considered, and the forces of the thrust bearing are neglected. The metal supporting structures that connect shafting system to concrete foundation, i.e. upper bracket, lower bracket and head cover, are assumed to be rigid laterally herein. If in some cases that deformations of the metal supporting structures are expected to be a consideration in the modeling, they may be made springs in series connected to the guide bearing models.

\subsection{Forces acting on shaft system}

\subsubsection{Damping}

When the vibration is generated under excitation, the structural damping loads acting on the rotor and turbine can be defined as [4]:

Rotor damping loads:

$$
\left\{\begin{array}{l}
F_{c x 1}=c_{1} \dot{x}_{1} \\
F_{c y 1}=c_{1} \dot{y}_{1}
\end{array}\right.
$$

Turbine damping loads:

$$
\left\{\begin{array}{l}
F_{c x 2}=c_{2} \dot{x}_{2} \\
F_{c y 2}=c_{2} \dot{y}_{2}
\end{array}\right.
$$

Where, $c_{1}$ and $c_{2}$ are the damping coefficients.

\subsubsection{Unbalanced magnetic pull}

Unbalanced magnetic pull results from air gap eccentricity, which can cause a potential risk to the dynamic stability of shafting system. Hence, correct analysis of hydropower unit vibration requires a load analysis involving the unbalanced magnetic pull. According to previous studies ${ }^{[4,19]}$, the unbalanced magnetic pull can be expressed as

$$
\begin{array}{r}
\left\{\begin{array}{c}
F_{x_{-} u m p} \\
F_{y_{-} u m p}
\end{array}\right\}=\frac{R L \pi K_{j}^{2} I_{j}^{2}}{4 \mu_{0}}\left(2 \Lambda_{0} \Lambda_{1}+\Lambda_{1} \Lambda_{2}+\Lambda_{2} \Lambda_{3}\right)\left\{\begin{array}{c}
\cos \gamma \\
\sin \gamma
\end{array}\right\} \\
\Lambda_{n}=\left\{\begin{array}{c}
\frac{\mu_{0} \frac{1}{\delta_{0}} \sqrt{1-\varepsilon^{2}}}{(n=0)} \\
\frac{2 \mu_{0}}{\delta_{0}} \frac{1}{\sqrt{1-\varepsilon^{2}}}\left[\frac{1-\sqrt{1-\varepsilon^{2}}}{\varepsilon}\right]^{n} \quad(n>0)
\end{array}\right.
\end{array}
$$

Where, $R, L$ are the radius and length of the rotor; $K_{j}$ is the coefficient of magnetomotive force fundamental wave of the air gap; $I_{j}$ is the exciting current of the generator; $\mu_{0}$ is the coefficient of the air gap permeance; $\Lambda_{n}$ are the Fourier coefficients of the air gap permeance; $\delta_{0}$ is uniform air gap length; $\varepsilon=$ $\frac{e}{\delta_{0}}$ is the relative eccentricity ( $e=\sqrt{x_{1}^{2}+y_{1}^{2}}$ is the radial displacement of rotor); $\gamma$ is the generator angle.

\subsubsection{Seal fluid excitation force}

The fluid induced vibration from seals is usually primary source of the system instability for hydro-turbine-generator units. The seal fluid excitation force can be written as Eq.(6) ${ }^{[20]}$ 


$$
\begin{gathered}
\left\{\begin{array}{c}
F_{x_{\text {_seal }}} \\
F_{y_{-} \text {seal }}
\end{array}\right\}=\left[\begin{array}{cc}
m_{f} \tau_{f}^{2} \omega^{2}-K & -\tau_{f} \omega D \\
\tau_{f} \omega D & m_{f} \tau_{f}^{2} \omega^{2}-K
\end{array}\right]\left\{\begin{array}{l}
x_{2} \\
y_{2}
\end{array}\right\}-\left[\begin{array}{cc}
D & 2 m_{f} \tau_{f} \omega \\
-2 m_{f} \tau_{f} \omega & D
\end{array}\right]\left\{\begin{array}{l}
\dot{x}_{2} \\
\dot{y}_{2}
\end{array}\right\}-\left[\begin{array}{cc}
m_{f} & 0 \\
0 & m_{f}
\end{array}\right]\left\{\begin{array}{l}
\ddot{x}_{2} \\
\ddot{y}_{2}
\end{array}\right\} \\
\left\{\begin{array}{c}
K=K_{0}\left(1-e^{2}\right)^{-n} \\
D=D_{0}\left(1-e^{2}\right)^{-n} \\
\tau_{f}=\tau_{0}(1-e)^{b}
\end{array}\right.
\end{gathered}
$$

Where, $K$ is the equivalent stiffness; $m_{f}, D$ are the equivalent quality and equivalent damping. And $K, D, \tau_{f}$ are the nonlinear functions of rotor lateral displacements $\left(x_{2}, y_{2}\right)$ at the disk. $e$ is the relatively eccentric distance of rotor; $c$ is the seal gap.

\subsubsection{Nonlinear oil-film forces}

The nonlinear oil-film forces are the major contributing factors which result in the hydro-turbine-generator units to be a self-exciting vibration and make unstable accidents. The oil-film instability would also lead to the significant nonlinear dynamic behavior of the shafting system varies with the rotational speed and model parameters. The nonlinear oil-film forces can be written as ${ }^{[21]}$

$$
\left\{\begin{array}{l}
F_{x_{-} y m}=\sigma \bar{f}_{x_{-} y m} \\
F_{y_{-} y m}=\sigma \bar{f}_{y_{-} y m}
\end{array}\right.
$$

where

$$
\begin{gathered}
\sigma=\mu \omega R_{b} L_{b}\left(\frac{R_{b}}{c_{z}}\right)^{2}\left(\frac{L_{b}}{2 R_{b}}\right)^{2} \\
\left\{\begin{array}{c}
\bar{f}_{x_{y m}} \\
\bar{f}_{y_{y m}}
\end{array}\right\}=\frac{\left[\left(x-2 y^{\prime}\right)^{2}+\left(y+2 x^{\prime}\right)^{2}\right]^{\frac{1}{2}}}{1-x^{2}-y^{2}} \cdot\left[\begin{array}{l}
3 x V(x, y, \alpha)-\sin \alpha G(x, y, \alpha)-2 \cos \alpha S(x, y, \alpha) \\
3 y V(x, y, \alpha)+\cos \alpha G(x, y, \alpha)-2 \sin \alpha S(x, y, \alpha)
\end{array}\right] \\
V(x, y, \alpha)=\frac{2+(y \cos \alpha-x \sin \alpha) G(x, y, \alpha)}{1-x^{2}-y^{2}} \\
S(x, y, \alpha)=\frac{x \cos \alpha+y \sin \alpha}{1-(x \cos \alpha+y \sin \alpha)^{2}} \\
G(x, y, \alpha)=\frac{2}{\left(1-x^{2}-y^{2}\right)^{\frac{1}{2}}} \cdot\left[\frac{\pi}{2}+\arctan \frac{y \cos \alpha-x \sin \alpha}{\left(1-x^{2}-y^{2}\right)^{\frac{1}{2}}}\right] \\
\alpha=\arctan \frac{y+2 x^{\prime}}{x-2 y^{\prime}}-\frac{\pi}{2} \operatorname{sgn}\left(\frac{y+2 x^{\prime}}{x-2 y^{\prime}}\right)-\frac{\pi}{2} \operatorname{sgn}\left(y+2 x^{\prime}\right)
\end{gathered}
$$

Where $\sigma$ is the correction factor of Sommerfeld; $\mu$ is the oil viscosity; $R_{b}$ is the radius of bearing; $L_{b}$ is the length of bearing; $c_{z}$ is the bearing radial clearance; $\bar{f}_{x_{-} y m}, \bar{f}_{y_{-} y m}$ are the non-dimension components of the oil-film forces.

\subsubsection{Modeling of the pulsating water pressure acting on turbine runner}

The pulsating water pressure acting on turbine runner is caused by the nonuniform water flow in pressure conduit of hydropower station. Most of the previous studies of shaft system only focused on the mechanical eccentric force and unbalanced sealing force, etc. Because there is lack of a mature method currently to determine the effect of water pressure fluctuation on the turbine runner vibration so that the influence of pressure fluctuations produced from the draft tube entrance and spiral case on the turbine runner is often simplified or ignored. Indeed, the behavior of the pulsating water pressure acting on turbine 
runner is often shown as the superposition of strong random uncertainty and harmonics, as shown in the typical measured data ${ }^{[22]}$ in Fig. 7.

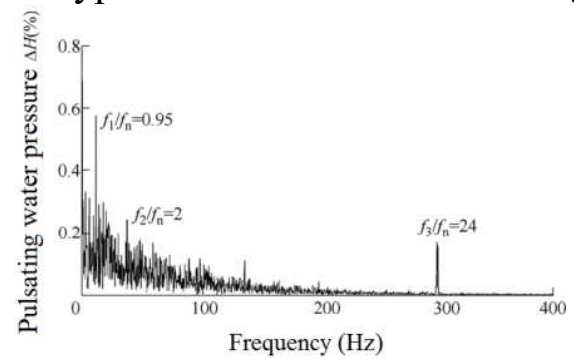

Fig. 7 Typical pressure fluctuation of the turbine runner tested on prototype machine [22]

In this study, based on a number of comprehensive investigations on the measured data, the pulsating water pressure acting on turbine runner is established in the following way:

$$
f(t)=\sum_{i=1}^{n} p_{i} \sin \left(\Omega_{i} t+\alpha_{i}\right)+f \xi(t)
$$

Where, $p_{i}$ is the amplitude of harmonic hydraulic excitation. $\Omega_{i}$ is the lower rotational frequency and its multiple frequencies, and it may also be the middle and higher electromagnetic vibration frequency and water pressure fluctuation $\left(50 \mathrm{~Hz}, 100 \mathrm{~Hz}\right.$, etc.). $\alpha_{i}$ is the random phase. $f$ is the coefficient of hydraulic excitation intensity. $\xi(t)$ is the random excitation and can be constructed by independent bounded noise [24].

$$
\xi(t)=A \sin \left[\omega_{0} t+\sigma B(t)+\gamma\right]
$$

where $A$ is the amplitude of noise; $\omega_{0}$ is the center frequency; $\sigma$ is the parameter of spectrum width; $B(\mathrm{t})$ is unit Wiener process; $\gamma$ is a random variable uniformly distributed in $[0,2 \pi) . \xi(t)$ is a stationary random process in wide sense with zero mean. Its covariance function is

$$
E\left[\xi\left(t_{1}\right) \xi\left(t_{2}\right)\right]=\frac{1}{2} \cos \omega_{0}\left(t_{1}-t_{2}\right) \cdot \exp \left(-\frac{\sigma^{2}}{2}\left|t_{1}-t_{2}\right|\right)
$$

and its spectral density is

$$
\mathrm{S}_{\xi \xi}(\Omega)=\frac{\sigma^{2}}{2 \pi}\left[\frac{1}{4\left(\Omega-\omega_{0}\right)^{2}+\sigma^{4}}+\frac{1}{4\left(\Omega+\omega_{0}\right)^{2}+\sigma^{4}}\right]
$$

which implies that the noise $\xi(\mathrm{t})$ is a generalized steady random process and has finite power. The shape of spectral density depends on $\omega_{0}, \sigma$ while the bandwidth of the noise depends mainly on $\sigma$. It is a narrow-band process when $\sigma$ is small and it approaches to white noise when $\sigma \rightarrow \infty$.

$\xi(\mathrm{t})$ is a reasonable model and can simulate effectively the random excitation or response in engineering systems by adjusting its parameters ( $\omega_{0}$ and $\left.\sigma\right)$. From $[24,25]$, each physical realization of bounded noise $\xi(\mathrm{t})$ can be approximated by

$$
\xi(t) \approx \sum_{k=1}^{N} A \cos \left(\omega_{o k} t+\phi_{k}\right) \quad(N \rightarrow \infty)
$$

Where, $A=\sqrt{2 S_{\xi \xi} \Delta \omega_{0}} \cdot\left\{\omega_{\mathrm{ok}} \mid k=1,2, \ldots, N\right\}$ are independent and nonnegative random variables over the interval $\left[\omega_{\mathrm{ol}}, \omega_{\mathrm{or}}\right],\left\{\phi_{\mathrm{k}} \mid k=1,2, \ldots, \mathrm{N}\right\}$ are identically uniformly distributed phases over the interval $[0,2 \pi) ; N$ is a large positive integer; $\Delta \omega_{0}=\left(\omega_{\mathrm{or}}-\omega_{\mathrm{ol}}\right) / N$ is a frequency increment. Therefore, the bounded noise $\xi(\mathrm{t})$ can be created as a deterministic function due to the approximated sum function. 


\subsection{Mathematical modeling of CSFS}

Use the notations T, $T_{1}$ and $T_{2}$ to denote the whole kinetic energy of CSFS, the kinetic energy of shafting system and the kinetic energy of foundation subsystem, respectively. Their relationship is given by

$$
\begin{aligned}
& \mathrm{T}=T_{1}+T_{2}=\frac{1}{2} m_{1}\left(\dot{x}_{1}^{2}+\dot{y}_{1}^{2}+e_{1}^{2} \dot{\phi}_{1}^{2}+2 e_{1} \dot{\phi}_{1} \dot{y}_{1} \cos \phi_{1}-2 e_{1} \dot{\phi}_{1} \dot{x}_{1} \sin \phi_{1}\right)+ \\
& \frac{1}{2}\left(J_{1}+m_{1} e_{1}^{2}\right) \dot{\phi}_{1}^{2}+\frac{1}{2} m_{2}\left(\dot{x}_{2}^{2}+\dot{y}_{2}^{2}+e_{2}^{2} \dot{\phi}_{2}^{2}+2 e_{2} \dot{\phi}_{2} \dot{y}_{2} \cos \phi_{2}-2 e_{2} \dot{\phi}_{2} \dot{x}_{2} \sin \phi_{2}\right)+ \\
& \frac{1}{2}\left(J_{2}+m_{2} e_{2}^{2}\right) \dot{\phi}_{2}^{2}+\frac{1}{2} m_{x 1} \dot{x}_{b 1}^{2}+\frac{1}{2} m_{x 2} \dot{x}_{b 2}^{2}+\frac{1}{2} m_{x 3} \dot{x}_{b 3}^{2}+\frac{1}{2} m_{y 1} \dot{y}_{b 1}^{2}+\frac{1}{2} m_{y 2} \dot{y}_{b 2}^{2}+ \\
& \frac{1}{2} m_{y 3} \dot{y}_{b 3}^{2}
\end{aligned}
$$

Where, $\left(x_{1}, y_{1}\right)$ and $\left(x_{2}, y_{2}\right)$ are the axis coordinates of the generator rotor and the turbine runner; $m_{1}, e_{1}, \phi_{1}, J_{1}$ are the quality, the mass eccentricity, the angle and rotational inertia of the generator rotor, respectively; $m_{2}, e_{2}, \phi_{2}, J_{2}$ are the quality, the mass eccentricity, the angle and rotational inertia of the turbine runner, respectively; $m_{x 1}, m_{x 2}, m_{x 3}, m_{y 1}, m_{y 2}, m_{y 3}$ are the lumped-masses of foundation subsystem in the $\mathrm{x}$ and $\mathrm{y}$ directions, respectively; $x_{b 1}, x_{b 2}, x_{b 3}, y_{b 1}, y_{b 2}, y_{b 3}$ are the coordinates of the lumped-masses in the $\mathrm{x}$ and $\mathrm{y}$ directions, respectively.

$A, B, C, O_{1}$ and $O_{2}$ are the centroids of the upper guide bearing, the lower guide bearing, the turbine guide bearing, the rotor of the generator and hydroturbine, as shown in Fig. 1(b). Set $\left|A O_{1}\right|=a,\left|O_{1} B\right|=b,|B C|=c,\left|C O_{2}\right|=d$, there will be

$$
\left\{\begin{array}{l}
r_{1}=\sqrt{x_{1}^{2}+y_{1}^{2}} \\
r_{2}=\sqrt{x_{2}^{2}+y_{2}^{2}} \\
r_{4}=\frac{(c+d) r_{1}+b r_{2}}{b+c+d} \\
r_{5}=\frac{d r_{1}+(b+c) r_{2}}{b+c+d}
\end{array}\right.
$$

where $r_{1}, r_{2}, r_{3}, r_{4}$ and $r_{5}$ are the radial displacements of the generator rotor, turbine runner, the upper guide bearing, the lower guide bearing and the turbine guide bearing, respectively; $A_{1}=(a+b)(b+c+d), A_{2}=\mathrm{ab}, \quad B=$ $b(b+c+d)$.

The potential energy of CSFS in both lateral directions can be represented as

$U_{x}=\frac{1}{2} k_{1}\left(r_{3} \cos \alpha-x_{b 1}\right)^{2}+\frac{1}{2} k_{2}\left(r_{4} \cos \alpha-x_{b 2}\right)^{2}+\frac{1}{2} k_{3}\left(r_{5} \cos \alpha-x_{b 3}\right)^{2}+$

$\frac{1}{2} k_{b x 1}\left(x_{b 1}-x_{b 2}\right)^{2}+\frac{1}{2} k_{b x 2}\left(x_{b 2}-x_{b 3}\right)^{2}+\frac{1}{2} k_{b x 3} x_{b 3}{ }^{2}$

$U_{y}=\frac{1}{2} k_{1}\left(r_{3} \sin \alpha-y_{b 1}\right)^{2}+\frac{1}{2} k_{2}\left(r_{4} \sin \alpha-y_{b 2}\right)^{2}+\frac{1}{2} k_{3}\left(r_{5} \sin \alpha-y_{b 3}\right)^{2}+$

$\frac{1}{2} k_{b y 1}\left(y_{b 1}-y_{b 2}\right)^{2}+\frac{1}{2} k_{b y 2}\left(y_{b 2}-y_{b 3}\right)^{2}+\frac{1}{2} k_{b y 3} y_{b 3}{ }^{2}$

The total potential energy of CSFS can be given by

$$
\begin{aligned}
& \mathrm{U}=U_{x}+U_{y}=\left[\frac{A_{1}^{2}}{B^{2}} k_{1}+\frac{(c+d)^{2}}{(b+c+d)^{2}} k_{2}+\frac{d^{2}}{(b+c+d)^{2}} k_{3}\right] \frac{x_{1}^{2}+y_{1}^{2}}{2}+\left[\frac{A_{2}^{2}}{B^{2}} k_{1}+\right. \\
& \left.\frac{b^{2}}{(b+c+d)^{2}} k_{2}+\frac{(b+c)^{2}}{(b+c+d)^{2}} k_{3}\right] \frac{x_{2}^{2}+y_{2}^{2}}{2}+\left[-\frac{A_{1} A_{2}}{B^{2}} k_{1}+\frac{b(c+d)}{(b+c+d)^{2}} k_{2}+\right.
\end{aligned}
$$




$$
\begin{aligned}
& \left.\frac{d(b+c)}{(b+c+d)^{2}} k_{3}\right] \sqrt{x_{1}^{2}+y_{1}^{2}} \sqrt{x_{2}^{2}+y_{2}^{2}}+\left(-\frac{A_{1}}{B} k_{1}\right)\left(x_{1} x_{b 1}+y_{1} y_{b 1}\right)+ \\
& \left(-\frac{c+d}{b+c+d} k_{2}\right)\left(x_{1} x_{b 2}+y_{1} y_{b 2}\right)+\left(-\frac{d}{b+c+d} k_{3}\right)\left(x_{1} x_{b 3}+y_{1} y_{b 3}\right)+ \\
& \left(\frac{A_{2}}{B} k_{1}\right)\left(x_{2} x_{b 1}+y_{2} y_{b 1}\right)+\left(-\frac{b}{b+c+d} k_{2}\right)\left(x_{2} x_{b 2}+y_{2} y_{b 2}\right)+ \\
& \left(-\frac{b+c}{b+c+d} k_{3}\right)\left(x_{2} x_{b 3}+y_{2} y_{b 3}\right)+\frac{1}{2}\left(k_{1}+k_{b x 1}\right) x_{b 1}^{2}+\frac{1}{2}\left(k_{2}+k_{b x 1}+k_{b x 2}\right) x_{b 2}^{2}+ \\
& \frac{1}{2}\left(k_{3}+k_{b x 2}+k_{b x 3}\right) x_{b 3}^{2}+\frac{1}{2}\left(k_{1}+k_{b y 1}\right) y_{b 1}^{2}+\frac{1}{2}\left(k_{2}+k_{b y 1}+k_{b y 2}\right) y_{b 2}^{2}+ \\
& \frac{1}{2}\left(k_{3}+k_{b y 2}+k_{b y 3}\right) y_{b 3}^{2}-k_{b x 1} x_{b 1} x_{b 2}-k_{b x 2} x_{b 2} x_{b 3}-k_{b y 1} y_{b 1} y_{b 2}- \\
& k_{b y 2} y_{b 2} y_{b 3}
\end{aligned}
$$

Based on the Lagrange equation, the dynamic equations of CSFS in the $\mathrm{x}$ direction can be written as Eqs. (18-1) (18-5). The difference between the x-direction and the y-direction is only the rotational phase, i.e., the "cos $\phi$ " in the x-direction should be replaced with " $\sin \phi$ " in the y-direction. The equations in y-direction are omitted for clarity.

$$
\begin{aligned}
& m_{1} \ddot{x}_{1}+c_{1} \dot{x}_{1}+\left[\frac{A_{1}^{2}}{B^{2}} k_{1}+\frac{(c+d)^{2}}{(b+c+d)^{2}} k_{2}+\frac{d^{2}}{(b+c+d)^{2}} k_{3}\right] x_{1}+\left[-\frac{A_{1} A_{2}}{B^{2}} k_{1}+\frac{b(c+d)}{(b+c+d)^{2}} k_{2}+\right. \\
& \left.\frac{d(b+c)}{(b+c+d)^{2}} k_{3}\right] \frac{\sqrt{x_{2}^{2}+y_{2}^{2}}}{\sqrt{x_{1}^{2}+y_{1}^{2}}} x_{1}-\frac{A_{1}}{B} k_{1} x_{b 1}-\frac{c+d}{b+c+d} k_{2} x_{b 2}-\frac{d}{b+c+d} k_{3} x_{b 3}=m_{1} e_{1} \omega^{2} \cos \phi+ \\
& F_{x_{-} u m p}+F_{x_{-} y m} \\
& m_{2} \ddot{x}_{2}+c_{2} \dot{x}_{2}+\left[\frac{A_{2}^{2}}{B^{2}} k_{1}+\frac{b^{2}}{(b+c+d)^{2}} k_{2}+\frac{(b+c)^{2}}{(b+c+d)^{2}} k_{3}\right] x_{2}+\left[-\frac{A_{1} A_{2}}{B^{2}} k_{1}+\frac{b(c+d)}{(b+c+d)^{2}} k_{2}+\right. \\
& \left.\frac{d(b+c)}{(b+c+d)^{2}} k_{3}\right] \frac{\sqrt{x_{1}^{2}+y_{1}^{2}}}{\sqrt{x_{2}^{2}+y_{2}^{2}}} x_{2}+\frac{A_{2}}{B} k_{1} x_{b 1}-\frac{b}{b+c+d} k_{2} x_{b 2}-\frac{b+c}{b+c+d} k_{3} x_{b 3}=m_{2} e_{2} \omega^{2} c o s \phi+ \\
& F_{x_{-} s e a l}+f_{x}(t) \\
& m_{x 1} \ddot{x}_{b 1}+c_{b x 1} \dot{x}_{b 1}+\left(k_{1}+k_{b x 1}\right) x_{b 1}-\frac{A_{1}}{B} k_{1} x_{1}+\frac{A_{2}}{B} k_{1} x_{2}-c_{b x 1} \dot{x}_{b 2}-k_{b x 1} x_{b 2}=0 \\
& m_{x 2} \ddot{x}_{b 2}+\left(c_{b x 1}+c_{b x 2}\right) \dot{x}_{b 2}+\left(k_{2}+k_{b x 1}+k_{b x 2}\right) x_{b 2}-\frac{c+d}{b+c+d} k_{2} x_{1}+\frac{b}{b+c+d} k_{2} x_{2}- \\
& c_{b x 1} \dot{x}_{b 1}-k_{b x 1} x_{b 1}-c_{b x 2} \dot{x}_{b 3}-k_{b x 2} x_{b 3}=0 \\
& m_{x 3} \ddot{x}_{b 3}+\left(c_{b x 2}+c_{b x 3}\right) \dot{x}_{b 3}+\left(k_{3}+k_{b x 2}+k_{b x 3}\right) x_{b 3}-\frac{d}{b+c+d} k_{3} x_{1}+\frac{b+c}{b+c+d} k_{3} x_{2}- \\
& c_{b x 2} \dot{x}_{b 2}-k_{b x 2} x_{b 2}=0
\end{aligned}
$$

\subsection{Deducing space state equations}

The length ratio of shaft system size is given by $a: b: c: d=3: 4: 6: 3$. The essential state variables $\left(z_{1}, z_{2}, \cdots, z_{20}\right)$ are specified in the solving procedure and listed in Eq. (19). Then, the higher-order dynamic equations stated above can be converted into first-order state equations (Eqs. (20-1) (20-10)).

$$
\left\{\begin{array}{c}
z_{1}=x_{1}, z_{2}=\dot{x}_{1}, \quad z_{3}=y_{1}, z_{4}=\dot{y}_{1}, \\
z_{5}=x_{2}, z_{6}=\dot{x}_{2}, \quad z_{7}=y_{2}, z_{8}=\dot{y}_{2}, \\
z_{9}=x_{b 1}, z_{10}=\dot{x}_{b 1}, \quad z_{11}=y_{b 1}, z_{12}=\dot{y}_{b 1}, \\
z_{13}=x_{b 2}, \quad z_{14}=\dot{x}_{b 2}, \quad z_{15}=y_{b 2}, z_{16}=\dot{y}_{b 2}, \\
z_{17}=x_{b 3}, \quad z_{18}=\dot{x}_{b 3}, \quad z_{19}=y_{b 3}, \quad z_{20}=\dot{y}_{b 3},
\end{array}\right.
$$




$$
\begin{aligned}
& \dot{z}_{1}=z_{2} \quad ; \quad \dot{z}_{2}=-\frac{1}{m_{1}}\left[c_{1} z_{2}+\left(\frac{49}{16} k_{1}+\frac{81}{169} k_{2}+\frac{9}{169} k_{3}\right) z_{1}+\left(-\frac{21}{52} k_{1}+\frac{36}{169} k_{2}+\frac{30}{169} k_{3}\right) \frac{\sqrt{z_{5}^{2}+z_{7}^{2}}}{\sqrt{z_{1}^{2}+z_{3}^{2}}} z_{1}-\frac{7}{4} k_{1} z_{9}-\right. \\
& \left.\frac{9}{13} k_{2} z_{13}-\frac{3}{13} k_{3} z_{17}\right]+e_{1} \omega^{2} \cos \phi+\frac{F_{x_{-} u m p}+F_{x_{-} y m}}{m_{1}} \\
& \dot{z}_{3}=z_{4} \quad ; \quad \dot{z}_{4}=-\frac{1}{m_{1}}\left[c_{1} z_{4}+\left(\frac{49}{16} k_{1}+\frac{81}{169} k_{2}+\frac{9}{169} k_{3}\right) z_{3}+\left(\frac{21}{52} k_{1}+\frac{36}{169} k_{2}+\frac{30}{169} k_{3}\right) \frac{\sqrt{z_{5}^{2}+z_{7}^{2}}}{\sqrt{z_{1}^{2}+z_{3}^{2}}} z_{3}-\frac{7}{4} k_{1} z_{11}-\right. \\
& \left.\frac{9}{13} k_{2} z_{15}-\frac{3}{13} k_{3} z_{19}\right]+e_{1} \omega^{2} \sin \phi+\frac{F_{y \_u m p}+F_{y_{\_} y m}}{m_{1}} \\
& \dot{z}_{5}=z_{6} \quad ; \quad \dot{z}_{6}=-\frac{1}{m_{2}+m_{f}}\left[\left(c_{2}+D\right) z_{6}+\left(\frac{9}{169} k_{1}+\frac{16}{169} k_{2}+\frac{100}{169} k_{3}\right) z_{5}+\left(-\frac{21}{52} k_{1}+\frac{36}{169} k_{2}+\frac{30}{169} k_{3}\right) \frac{\sqrt{z_{1}^{2}+z_{3}^{2}}}{\sqrt{z_{5}^{2}+z_{7}^{2}}} z_{5}+\right. \\
& \left(k-m_{f} \tau_{f}^{2} \omega^{2}\right) z_{5}+\tau_{f} \omega D z_{7}+2 \tau_{f} m_{f} \omega z_{8}+\frac{3}{13} k_{1} z_{9}-\frac{4}{13} k_{2} z_{13}-\frac{10}{13} k_{3} z_{17}+m_{2} e_{2} \omega^{2} \cos \phi+ \\
& \left.f_{x}(t)\right] \\
& \dot{z}_{7}=z_{8} \quad ; \quad \dot{z}_{8}=-\frac{1}{m_{2}+m_{f}}\left[\left(c_{2}+D\right) z_{8}+\left(\frac{9}{169} k_{1}+\frac{16}{169} k_{2}+\frac{100}{169} k_{3}\right) z_{7}+\left(-\frac{21}{52} k_{1}+\frac{36}{169} k_{2}+\frac{30}{169} k_{3}\right) \frac{\sqrt{z_{1}^{2}+z_{3}^{2}}}{\sqrt{z_{5}^{2}+z_{7}^{2}}} z_{7}+\right. \\
& \left.\left(k-m_{f} \tau_{f}^{2} \omega^{2}\right) z_{7}-\tau_{f} \omega D z_{5}-2 \tau_{f} m_{f} \omega z_{6}+\frac{3}{13} k_{1} z_{11}-\frac{4}{13} k_{2} z_{15}-\frac{10}{13} k_{3} z_{19}-m_{2} e_{2} \omega^{2} \sin \phi+f_{x}(t)\right] \\
& \dot{z}_{9}=z_{10} ; \dot{z}_{10}=-\frac{1}{m_{x 1}}\left[c_{b x 1} z_{10}+\left(k_{1}+k_{b x 1}\right) z_{9}-\frac{7}{4} k_{1} z_{1}+\frac{3}{13} k_{1} z_{5}-c_{b x 1} z_{14}-k_{b x 1} z_{13}\right] \\
& \dot{z}_{11}=z_{12} ; \dot{z}_{12}=-\frac{1}{m_{y 1}}\left[c_{b y 1} z_{12}+\left(k_{1}+k_{b y 1}\right) z_{11}-\frac{7}{4} k_{1} z_{3}+\frac{3}{13} k_{1} z_{7}-c_{b y 1} z_{16}-k_{b y 1} z_{15}\right] \\
& \dot{z}_{13}=z_{14} \quad ; \quad \dot{z}_{14}=-\frac{1}{m_{x 2}}\left[\left(c_{b x 1}+c_{b x 2}\right) z_{14}+\left(k_{2}+k_{b x 1}+k_{b x 2}\right) z_{13}-\frac{9}{13} k_{2} z_{1}-\frac{4}{13} k_{2} z_{5}-c_{b x 1} z_{10}-k_{b x 1} z_{9}-\right. \\
& \left.c_{b x 2} z_{18}-k_{b x 2} z_{17}\right] \\
& \dot{z}_{15}=z_{16} ; \quad \dot{z}_{16}=-\frac{1}{m_{y 2}}\left[\left(c_{b y 1}+c_{b y 2}\right) z_{16}+\left(k_{2}+k_{b y 1}+k_{b y 2}\right) z_{15}-\frac{9}{13} k_{2} z_{3}-\frac{4}{13} k_{2} z_{7}-c_{b y 1} z_{12}-k_{b y 1} z_{11}-\right. \\
& \left.c_{b y 2} z_{20}-k_{b y 2} z_{19}\right] \\
& \dot{z}_{17}=z_{18} ; \dot{z}_{18}=-\frac{1}{m_{x 3}}\left[\left(c_{b x 2}+c_{b x 3}\right) z_{18}+\left(k_{3}+k_{b x 2}+k_{b x 3}\right) z_{17}-\frac{3}{13} k_{3} z_{1}-\frac{10}{13} k_{3} z_{5}-c_{b x 2} z_{14}-k_{b x 2} z_{13}\right] \\
& \dot{z}_{19}=z_{20} \quad ; \quad \dot{z}_{20}=-\frac{1}{m_{y 3}}\left[\left(c_{b y 2}+c_{b y 3}\right) z_{20}+\left(k_{3}+k_{b y 2}+k_{b y 3}\right) z_{19}-\frac{3}{13} k_{3} z_{3}-\frac{10}{13} k_{3} z_{7}-c_{b y 2} z_{16}-\right. \\
& \left.k_{b y 2} z_{15}\right]
\end{aligned}
$$

\section{Dynamic simulation of CSFS and validation}

On the basis of the energy-equivalent nonlinear vibration model developed for CSFS, the prototype mechanical behavior of hydropower station located in southwest of China is simulated and discussed here. The coupled nonlinear vibration model is developed and analyzed using adaptive step size fourth-fifth order Runge-Kutta method of the MATLAB software. Meanwhile, the results of the corresponding field tests are used to validate the equivalent simplified model of CSFS in this paper. The main relevant parameters of the CSFS are as follows:

The hydropower house has five Francis hydro generator units installed, each with $350 \mathrm{MW}$ and of semi-umbrella vertical shaft type. The concrete material of the units foundation is $\mathrm{C} 25$. The spacing between units is $34.3 \mathrm{~m}$. The length sizes 
of shafting are $\left|A O_{1}\right|=a=3.75 \mathrm{~m},\left|O_{1} B\right|=b=5 \mathrm{~m},|B C|=c=7.5 \mathrm{~m}$, $\left|C O_{2}\right|=d=3.75 \mathrm{~m}$. The masses of the generator rotor and water turbine runner are $m_{1}=2.2 \times 10^{6} \mathrm{~kg}$ and $m_{2}=2.21 \times 10^{6} \mathrm{~kg}$. The damping coefficients of the generator rotor and hydraulic turbine runner are $c_{1}=3.0 \times 10^{4} \mathrm{~N} \cdot \mathrm{s} / \mathrm{m}, c_{2}=4.0 \times 10^{4} \mathrm{~N} \cdot \mathrm{s} / \mathrm{m}$. The upper guide bearing stiffness is $k_{1}=2.5 \times 10^{9} \mathrm{~N} / \mathrm{m}$; the lower guide bearing stiffness is $k_{2}=0.9 \times 10^{9} \mathrm{~N} / \mathrm{m}$; the turbine guide bearing stiffness is $k_{3}=0.8 \times 10^{9} \mathrm{~N} / \mathrm{m}$. The mass eccentricities of the generator rotor and turbine runner are $e_{1}=5.0 \times 10^{-4} \mathrm{~m}$ and $e_{2}=3.0 \times 10^{-4} \mathrm{~m}$. The radius and length of generator rotor are $R_{\mathrm{r}}=9 \mathrm{~m}$ and $L_{\mathrm{r}}=4 \mathrm{~m}$. The uniform air gap is $\delta_{0}=2.9 \times 10^{-2} \mathrm{~m}$. The air permeability is $\mu_{0}=4 \pi \times 10^{-7} \mathrm{H} / \mathrm{m}$. The air gap flux potential is $k_{\mathrm{j}}=5.1$. The generator excitation current is $I_{j}=700 \mathrm{~A} . n, b$ and $\tau_{0}$ are used to describe the specific seal: $n=2.5 、 b=0.2 、 \tau_{0}=0.5 . n_{0}$ and $m_{0}$ are the empirical coefficients determined by the experiment and seal structures: $n_{0}=0.079$ 、 $m_{0}=-0.250$. The local loss coefficient is $\xi=1.5$. Seal radius and seal length are $R_{\mathrm{s}}=3.0 \mathrm{~m} 、 L_{\mathrm{s}}=0.5 \mathrm{~m}$. The axial velocity is $v=3.0 \mathrm{~m} / \mathrm{s}$. Hydrodynamic viscosity coefficient is $\gamma=1.3 \times 10^{-3} \mathrm{~Pa} \cdot \mathrm{s}$. The sealing pressure drop is $\Delta \mathrm{P}=5.0 \times 10^{5} \mathrm{~Pa}$. The seal clearance is $c=2.0 \times 10^{-3} \mathrm{~m}$. Lubricating oil viscosity is $\mu=2.4 \times 10^{-2} \mathrm{H} / \mathrm{m}$. Bearing radius clearance is $c_{z}=4.0 \times 10^{-4} \mathrm{~m}$. Radius of bearing is $R_{b}=0.3 \mathrm{~m}$. Bearing length is $L_{b}=0.5 \mathrm{~m}$. Rated unit speed is $\omega=7.85 \mathrm{rad} / \mathrm{s}$. Runaway speed of the turbine is $15.7 \mathrm{rad} / \mathrm{s}$.

\subsection{Brief description of field experimental tests}

Song and Ma [26] made a detailed study on the prototype coupled-vibration tests of generator set and power-house. The measuring sensors are distributed in four areas including block concrete structure (i.e., the supporting foundation subsystem), the floor structure, water turbine generator set structure and the water pressure fluctuation measuring area in the draft tube and spiral case. The detailed testing point arrangement is shown in Fig. 8.

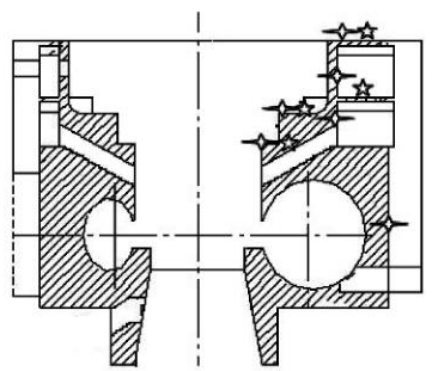

(a) Foundation subsystem

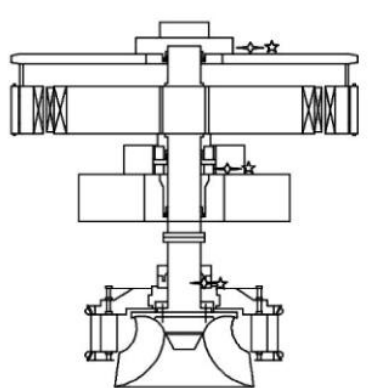

(b) Shaft subsystem

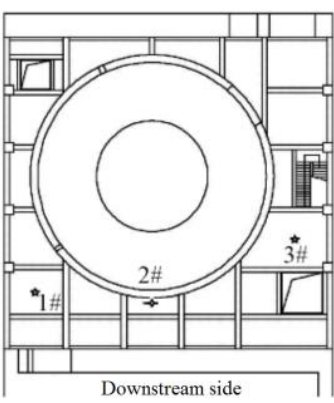

(c) Generator floor

Fig. 8 Sketch of testing point arrangement [26]

The amplitudes of measured shaft swing during the load increasing phase are shown in Table. 2. The trend analysis indicates that the shaft swing decreases rapidly with the increase of the unit load. At rated load, the turbine bearing amplitude in lateral-river vibration is $267.7 \mu \mathrm{m}$.

Table 2 Amplitudes of measured shaft swing ( $\mu \mathrm{m})$ [26]

\begin{tabular}{ccccccc}
\hline Direction & Testing point & $50 \mathrm{MW}$ & $87.5 \mathrm{MW}$ & $175 \mathrm{MW}$ & $262.5 \mathrm{MW}$ & $350 \mathrm{MW}$ \\
\hline Lateral-river & $\begin{array}{c}\text { Turbine } \\
\text { bearing }\end{array}$ & $/$ & $/$ & 527.4 & 286.8 & 267.7 \\
& & & & & \\
Lateral-river & Upper bearing & 223.9 & 188.5 & 183.3 & 141.8 & 142.0 \\
Longitude-river & Upper bearing & 234.3 & 366.2 & 202.0 & 158.5 & 141.4 \\
\hline
\end{tabular}

For the foundation subsystem testing, the maximum effective amplitudes of lower bracket foundation in longitude-river direction and in vertical direction are $28 \mu \mathrm{m}$ and $56 \mu \mathrm{m}$, respectively. The dynamic response curves of foundation as the unit load 
increases are detailed in Fig. 9. Fig. 9 shows the vibration law of each part of the foundation is similar to that of the unit. The larger vibration of foundation subsystem occurs in the lower load area. When the unit output is $87.5 \mathrm{MW}$, the vibration of each part is the most intense, and the vibration gradually decreases with increasing load. Additional details on the test site, test method and analysis results can be found in the work by Song et al. [26]. These field tested results will be utilized to compare to the following simulated results and to validate the developed energy-equivalent model of CSFS

\subsection{Result discussion and model validation}

Compared to the traditional water turbine generator set shaft system model, the major advantage of the developed energy-equivalent model of CSFS in this paper is reflecting the effects of the supporting foundation on the unit vibration and the dynamic coupled relationship between the supporting foundation and the shafting system. To verify the energy-equivalent model of CSFS, the corresponding theoretical simulations using the established coupled dynamics model need be performed under the same pulsating water pressure conditions as the experimental tests. Hence, the primary steps in performing the verifying analysis are summarized in the following steps.

(1) Read the measured vibration data of shaft subsystem in the field experiments under different load cases.

(2) To obtain the equivalent mathematical model of CSFS, the equivalent pulsating water pressures acting on turbine runner corresponding to different load cases are identified using the data in step 1.

(3) The simulated vibration responses of the foundation subsystem in the equivalent simplified model of CSFS are extracted and compared to the field experimental tested results of foundation for model validation.

Comparisons between the tested and the simulated vibration acceleration results are made in Fig. 9. It is obviously observed that the acceleration root mean square values of calculated results have basically the similar variation law as field tested results under different unit loads. The values of the computed results obtained by the established model are also very close to the tested data in Song et al. [25]. Only relatively minor differences are observed on the acceleration results, which may be induced by the model itself simplification and the position deviations of the actual measuring points. Hence, the accuracy of optimal equivalent model for evaluating the foundation responses are deemed accurate enough for capturing the dynamic coupling relationship between the shafting subsystem and the foundation subsystem.

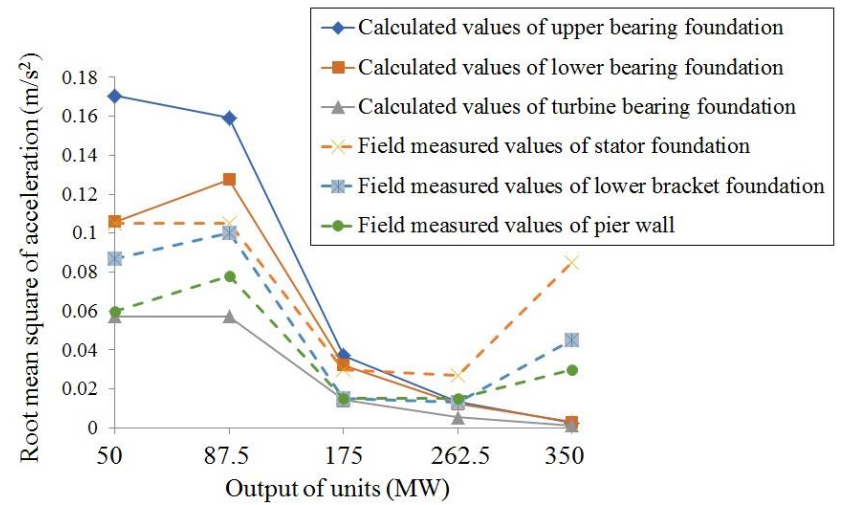

Fig. 9 Comparison of acceleration root mean square of foundation longitude-river vibration calculated by proposed CSFS model with field tested results in Song et al. [26].

In addition, some typical computed results are shown in Fig. 10 and Fig. 11, 
including the phrase diagram of the axis coordinates and foundation time-domain responses. It can be seen that the chaotic running state of the shafting system occurs in the different load cases, and the phrase diagrams of the axis coordinate under low-load condition are more unstable and have larger amplitudes than those under rated-load condition. In fact, the unit running state, i.e., it is chaos or quasi-periodic motion, mainly depends on excitation intensity and frequency band features of the constructed pulsating water pressure acting on turbine runner.

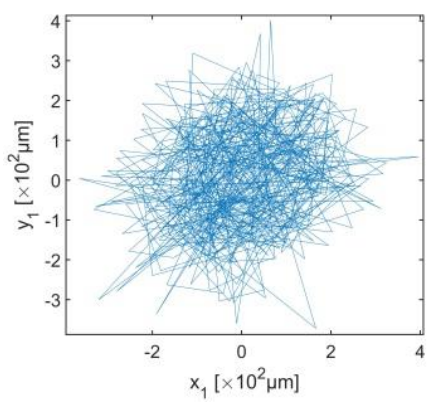

(a)

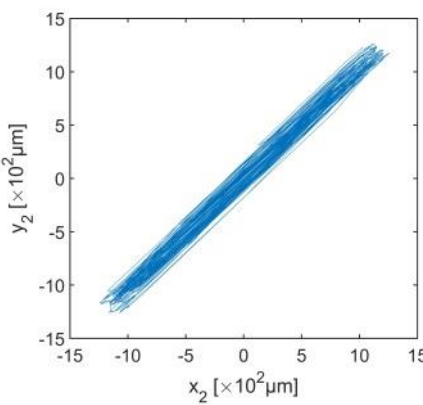

(b)

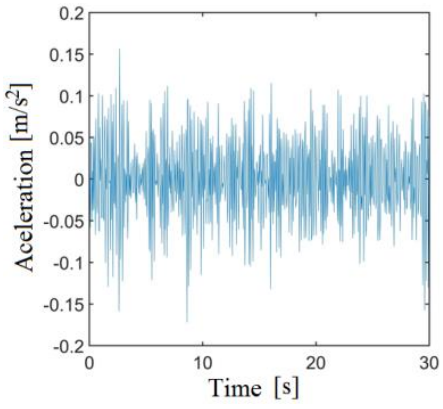

(c)

Fig. 10 Calculated vibration results of the CSFS in the case of 87.5MW load. (a) Phrase diagram of the axis coordinates for generator rotor; (b) Phrase diagram of the axis coordinates for turbine runner; (c) Aceleration-time history of lower bearing foundation.

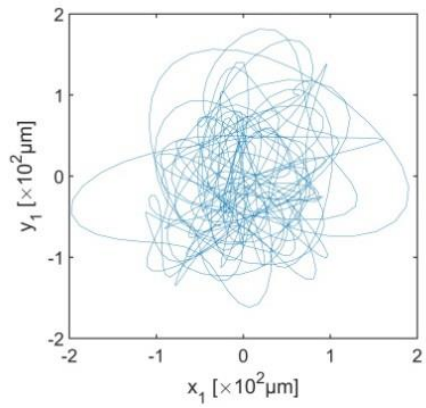

(a)

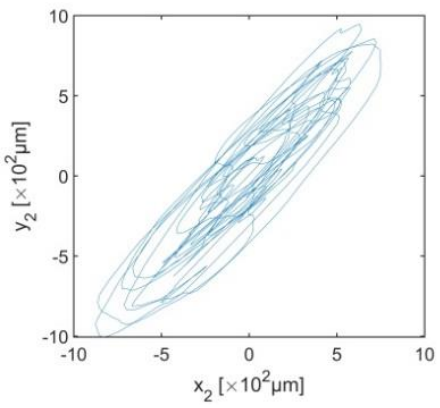

(b)

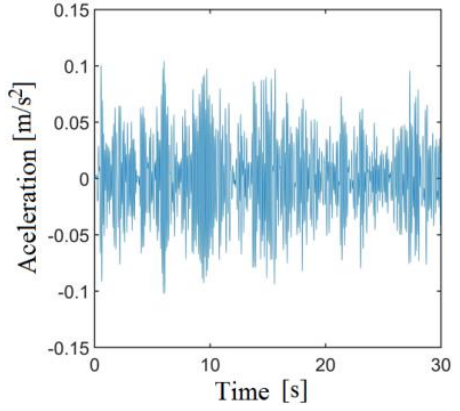

(c)

Fig. 11 Calculated vibration results of the CSFS in the case of 350MW load. (a) Phrase diagram of the axis coordinates for generator rotor; (b) Phrase diagram of the axis coordinates for turbine runner; (c) Aceleration-time history of lower bearing foundation.

\section{Effect of foundation in the nonlinear vibration of shaft system}

Previously published water turbine generator set shaft dynamics model with rigid supports did not consider the dynamic effects from the elastically supporting foundations and the mutually foundations coupling. Thus, the vibrations caused by excitations from the foundation subsystem cannot be revealed by using the traditional shaft dynamics model. In order to investigate the dynamic influence of the foundation subsystem, this section compares the nonlinear vibration characteristics from the established optimal equivalent model of CSFS and the individual hydro-generator shaft model without coupling foundations in the same rotational speed range from 0 to $20 \mathrm{rad} / \mathrm{s}$. Due to the abovementioned pulsating water pressure in the simplified model being an equivalent load, it is different and uncertain for different models at various rotational speeds. Hence, the complicated pulsating water pressure is neglected in this comparison.

The shaft lateral vibration results calculated by the two dynamics models are presented in Fig. 12 Fig. 14. Fig.12(a) and (b) show plots of vibrational 
bifurcation diagrams of the generator rotor and turbine runner for CSFS and individual shaft system. It is seen that as the rotational speed and thus also the radial amplitude of shaft system is increased. The amplitudes of CSFS are also generally higher than those of individual shaft system. The first peak of the critical speed of CSFS appears slightly earlier than that of individual shaft system, which is induced by the reduced support stiffness when considering foundation subsystem. In the whole operating speed range, there are always complex quasi periodic motions in the generator rotor of individual shaft system, in contrast to the stable periodic motions occurred in CSFS. The corresponding axial trajectory diagrams are depicted in Fig.13. The axial trajectories of rotor and runner in CSFS always appear as very regular circles at different rotational speeds, but the axial trajectories of rotor in individual shaft system appear as complex symmetrical rings. It is evident that the dynamic behavior of CSFS is completely different from that of the individual shaft system at lower rotating speeds.

The vibrational differences between the two structures are also reflected in the spectrum diagrams, as shown in Fig.14, which also reveal the inner reasons for these differences. Actually, the foundation subsystem is also used to provide additional damping to the shaft system besides elastic supporting. The effect of damping is to absorb vibration energy and to cause an obvious decrease in highfrequency motion of the shaft system. Meanwhile, due to the effect of lowfrequency vibrational behavior of the coupling foundation subsystem, the shaft subsystem in CSFS has also produced the obvious low-frequency amplification. These similar variations for frequency response characteristic can be found in other coupling dynamic system, such as the coupled vehicle-track system [27], etc.

In general, the nonlinear vibration of water turbine generator set should be analyzed as a whole coupling system, taking into account the foundation subsystem impact. The effect of foundation involves the amplitude characteristics, frequency characteristics and stability.

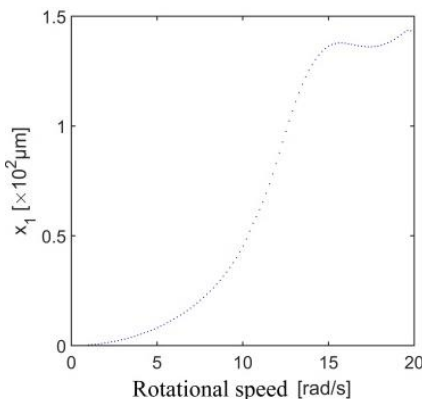

(a) Coupled shaft-foundation system

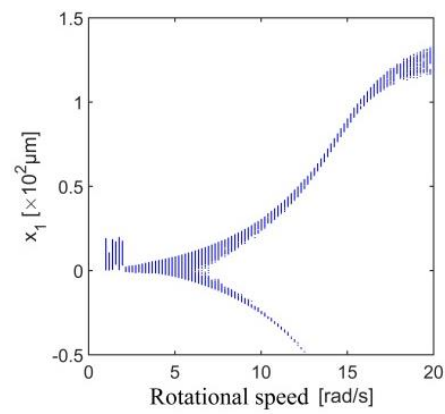

(b) Individual shaft system

Fig. 12(a) Bifurcation diagrams of generator rotor axis coordinate at different rotational speeds

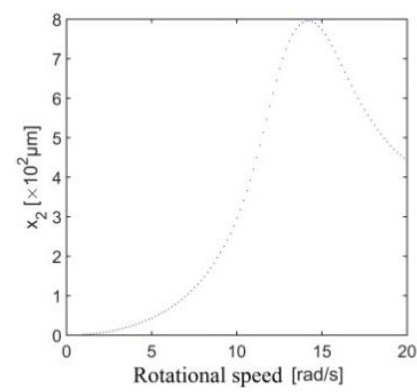

(a) Coupled shaft-foundation system

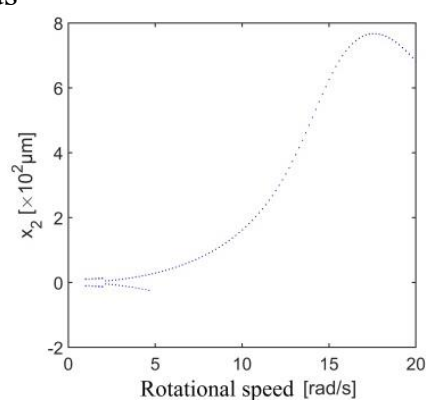

(b) Individual shaft system

Fig. 12(b) Bifurcation diagrams of turbine runner axis coordinate at different rotational speeds 


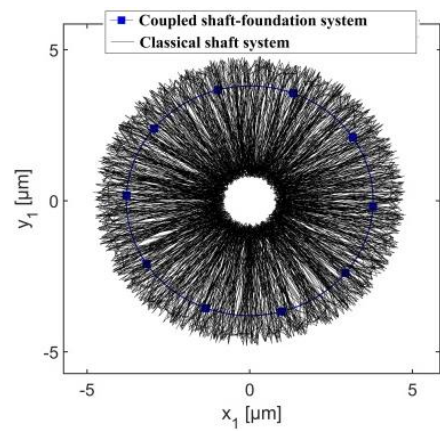

(a) $\omega=3.5 \mathrm{rad} / \mathrm{s}$

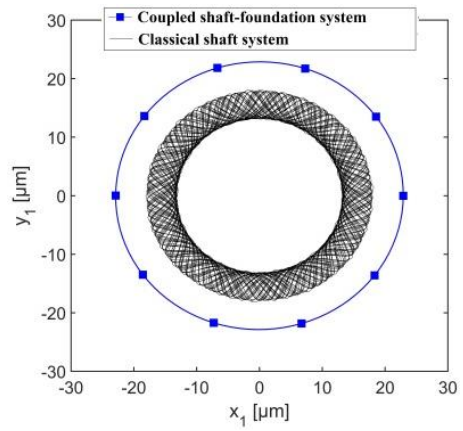

(b) $\omega=7.85 \mathrm{rad} / \mathrm{s}$

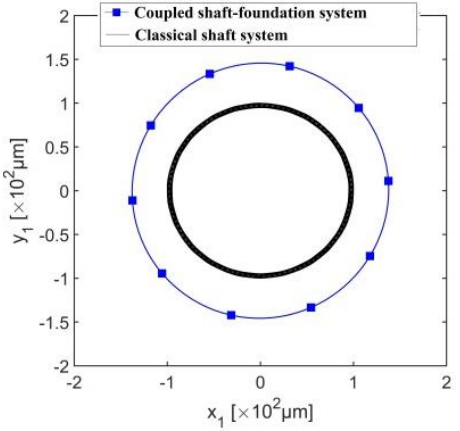

(c) $\omega=15.7 \mathrm{rad} / \mathrm{s}$

Fig. 13(a) Phrase diagrams of the axis coordinates for generator rotor at different rotational speeds

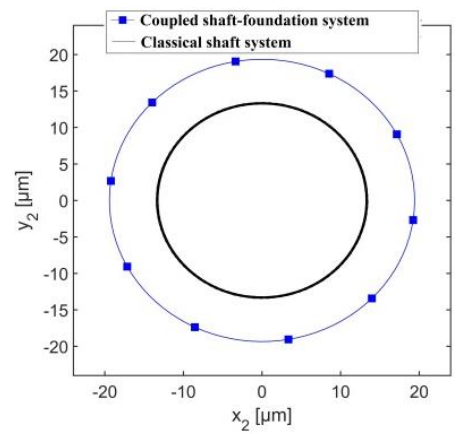

(a)

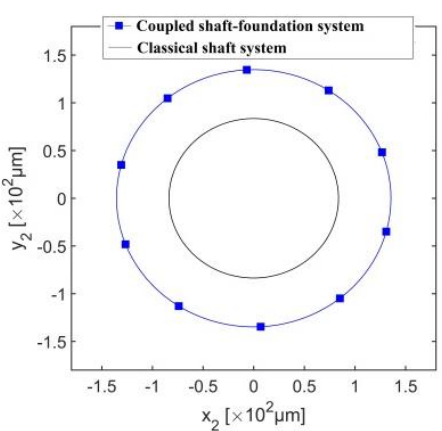

(b) $\omega=7.85 \mathrm{rad} / \mathrm{s}$

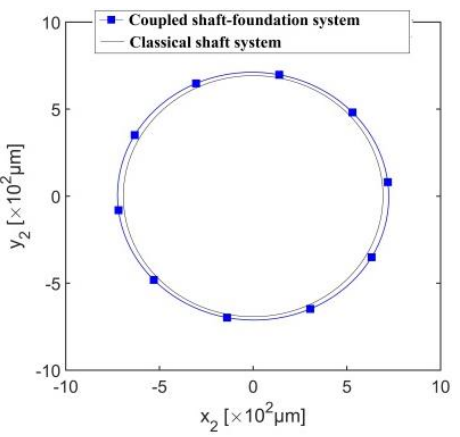

(c) $\omega=15.7 \mathrm{rad} / \mathrm{s}$

Fig. 13(b) Phrase diagrams of the axis coordinates for turbine runner at different rotational speeds

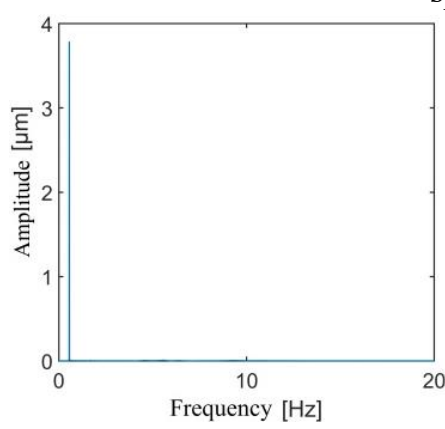

(a) Coupled shaft-foundation system

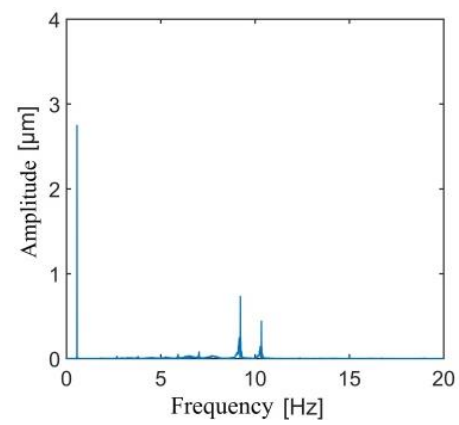

(b) Individual shaft system

Fig. 14(a) Amplitude-frequency figures of generator rotor lateral vibration $(\omega=3.5 \mathrm{rad} / \mathrm{s})$

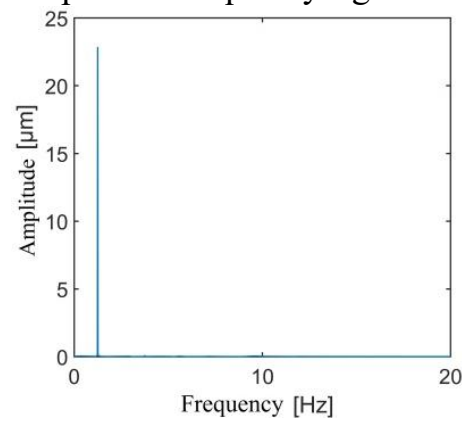

(a) Coupled shaft-foundation system

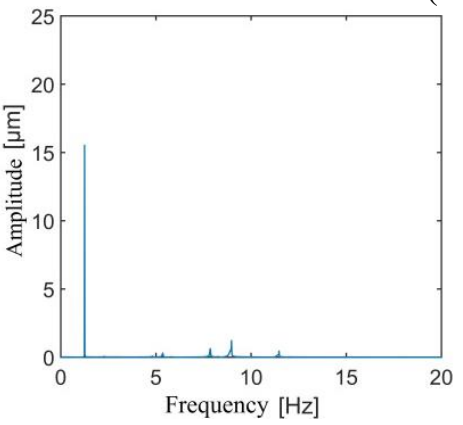

(b) Individual shaft system

Fig. 14(b) Amplitude-frequency figures of generator rotor lateral vibration $(\omega=7.85 \mathrm{rad} / \mathrm{s})$

\section{Conclusions}

In this article, a new energy-equivalent simplified model for lateral nonlinear vibrations of CSFS was established. The calculation of nonlinear dynamic responses of the CSFS was carried out using Runge-Kutta method in MATLAB. 
The mutual coupling responses of the shaft system and the foundation system were analyzed. This energy-equivalent simplified dynamics model enables the simulated dynamic responses of the CSFS close to the actual situation, which is also verified by comparing the calculated results with the tested data. Besides, due to fewer degrees of freedom, simpler modeling process and faster solving capability on the nonlinear dynamic performance of the CSFS are major advantages of the proposed coupled dynamics model. It can thus be further applied for analyzing the deeper dynamic performances of CSFS in the future work, such as sensitivity analyses and comparison of a large number of similar schemes, etc.

\section{Acknowledgments}

This work was supported by the National Key Research \& Development Project

(2016YFC0401900).

Conflict of Interest: The authors declare that they have no conflict of interest.

\section{References}

[1] Yong Xu, Zhaohui Li, Xide Lai, Dynamic model for hydro-turbine generator units based on a database method for guide bearings, Shock and Vibration. 20 (2013) 411-421.

[2] R. Cardinali, R. Nordmann, A. Sperber, Dynamic simulation of non-linear models of hydroelectric machinery, Mechanical Systems and Signal Processing. 7 (1993) 29-44.

[3] Huang Z , Zhou J , Yang M, et al., Vibration characteristics of a hydraulic generator unit rotor system with parallel misalignment and rub-impact, Archive of Applied Mechanics. 81 (2011) 829-838.

[4] Xu B, Chen D, Zhang H, et al., Dynamic analysis and modeling of a novel fractional-order hydro-turbine-generator unit, Nonlinear Dynamics. 81 (2015) 1263-1274.

[5] Lai Xide, Zhu Yi, Liao Gonglei, Lateral Vibration Response Analysis on Shaft System of Hydro Turbine Generator Unit, Advances in Vibration Engineering. 12 (2013) 511-524.

[6] Ma, Z.Y., Song, Z.Q., Nonlinear Dynamic Characteristic Analysis of the Shaft System in Water Turbine Generator Set, Chinese Journal of Mechanical Engineering. 22 (2009) 124-131.

[7] Karlsson, Martin, Aidanpaa, Jan-Olov, Dynamic behaviour in a hydro power rotor system due to the influence of generator shape and fluid dynamics, Proceedings of the ASME Power Conference 2005, Pts A and B, (2005)905-913.

[8] Sun, W.Q., Yan, D.M., Identification of the Nonlinear Vibration Characteristics in Hydropower House Using Transfer Entropy, Nonlinear Dynamics. 75 (2014) 673-691.

[9] Sun, W.Q., Ma, Z.Y., et al., Intelligent identification of underground powerhouse of pumpedstorage power plant, Acta Mech. Sin. 21 (2005) 187-191.

[10] Shen, X.; Jia, J.; Zhao, M.; Jing, J., Experimental and numerical analysis of nonlinear dynamics of rotor-bearing-seal system[J]. Nonlinear Dyn. 53 (2008)31-44

[11] Wang, Y., Dynamic response analysis of cracked hydropower house and spiral case. Master thesis (2006). Dalian University of Technology.

[12] Yao, Dechen, Nonlinear Dynamics of Rotating Machinery, Beijing institute of technology press, Beijing , 2017. 
[13] Qianqian $\mathrm{Wu}$, Leike Zhang, Zhenyue Ma, A model establishment and numerical simulation of dynamic coupled hydraulic-mechanical-electric-structural system for hydropower station, Nonlinear Dynamics. 87 (2017) 459-474.

[14] Leike Zhang, Qianqian $\mathrm{Wu}$, Zhenyue Ma, et al. Transient vibration analysis of unit-plant structure for hydropower station in sudden load increasing process. Mechanical Systems and Signal Processing 120 (2019) 486-504.

[15] Liaojun Zhang, Shuo Wang, Guojiang Yin, et al. Vibration transmission path identification in a hydropower house based on a time-delayed transfer entropy method, Journal of Vibration and Control. (2020), 26(15-16):107754631989481.

[16] Song Zhiqiang, MA Zhenyue, Zhang Yunliang, et al., Dynamic response analysis of rotorbearing system of a generator set accounting for whole hydropower house foundation, Journal of vibration and shock. 27 (2008) 158-161.

[17] Shi-Shuenn Chen; Jun-Yang Shi, A simplified model for coupled horizontal and rocking vibrations of embedded foundations, Soil Dynamics and Earthquake Engineering. 48 (2013) 209219.

[18] Lian,J., Tian,H.,Qin, L.,Zhang,Y., The method to identify dynamic characteristics of hydro plants supporting structure using machine halting process, Eng. Sci. 8(2006) 73-75.

[19] Zhou Jianzhong, Zhang Yongchuan, Li Chaoshun, Dynamics of Hydrogenerator Sets and Principles and Methods of Fault Diagnosis, Huazhong University of Science and Technology Press, 2013.

[20] Agnieszka Muszynska, Rotordynamics, CRC Press. Taylor \& Francis Group. 2005.

[21] Adiletta G, Guido A R, Rossi C, Chaotic motions of a rigid rotor in short journal bearings, Nonlinear Dynamics. 10 (1996) 251-269.

[22] LIU Demin; DENG Xiangping; ZHAO Yongzhi; et al., Dynamic Stress and Pressure Fluctuation Test on Model Unit of Huge Francis Turbine, Journal of mechanical engineering. 55 (2019) 9-18

[23] Lin Y K, Cai G Q, probabilistic structural dynamics: advanced theory and applications, New York: McGraw Hill Press, 2004.

[24] M. Shinozuka, C. M. Jan, Digital simulation of random processes and its applications, Journal of Sound and Vibration. 25 (1972) 111-128.

[25] Chunbiao Gan, Qingyun Wang, Matjaž Perc, Torus breakdown and noise-induced dynamics in the randomly driven Morse oscillator, Journal of Physics A: Mathematical and Theoretical. 43 (2010)125102.1-125102.13.

[26] SONG Zhiqiang, MA Zhenyue, Prototype coupled-vibration test of generator set and powerhouse and FEM feedback analysis. Journal of hydroelectric engineering. 31 (2012) 170-174, 205

[27] Wanming Zhai; Kaiyun Wang; Chengbiao Cai, Fundamentals of vehicle-track coupled dynamics, Vehicle System Dynamics. 47 (2009) 1349-1376. 


\section{Figures}

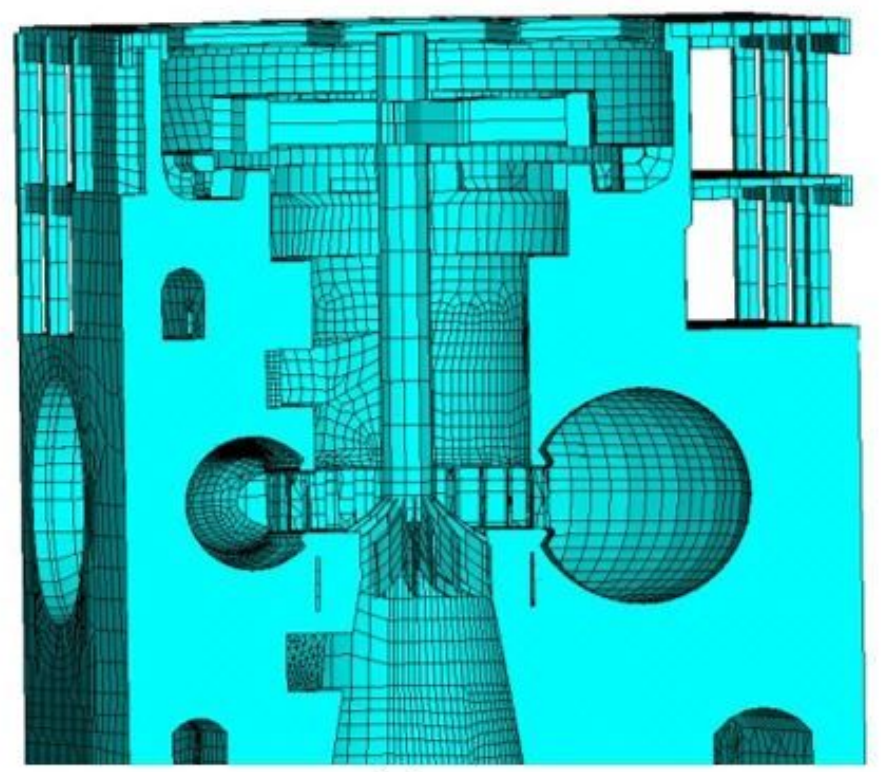

(a)

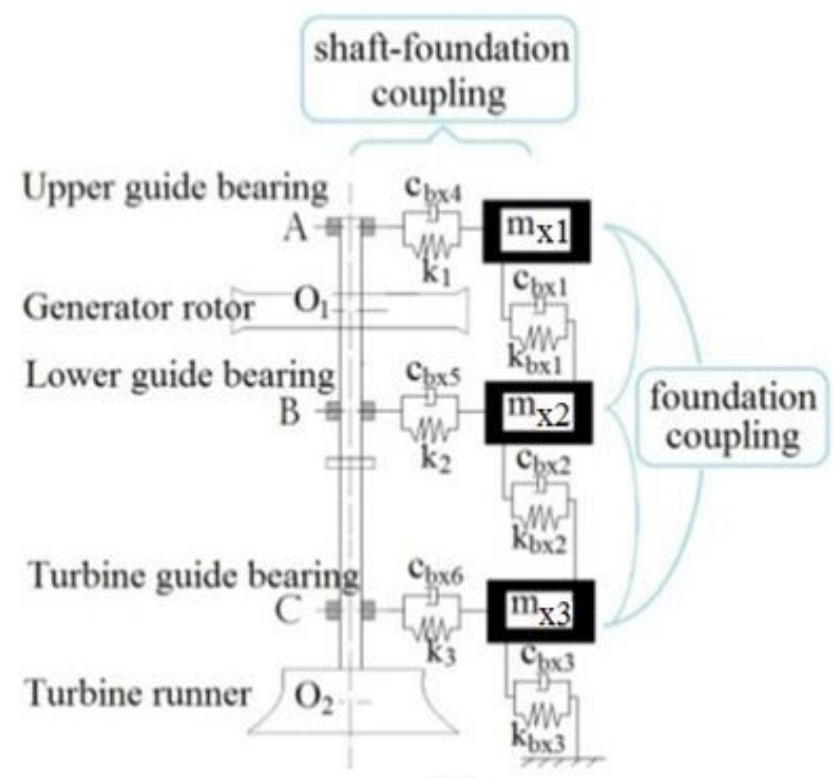

(b)

\section{Figure 1}

(a) Whole finite element model and (b) equivalent simplified model of a coupled water turbine generator set shaft-foundation system 


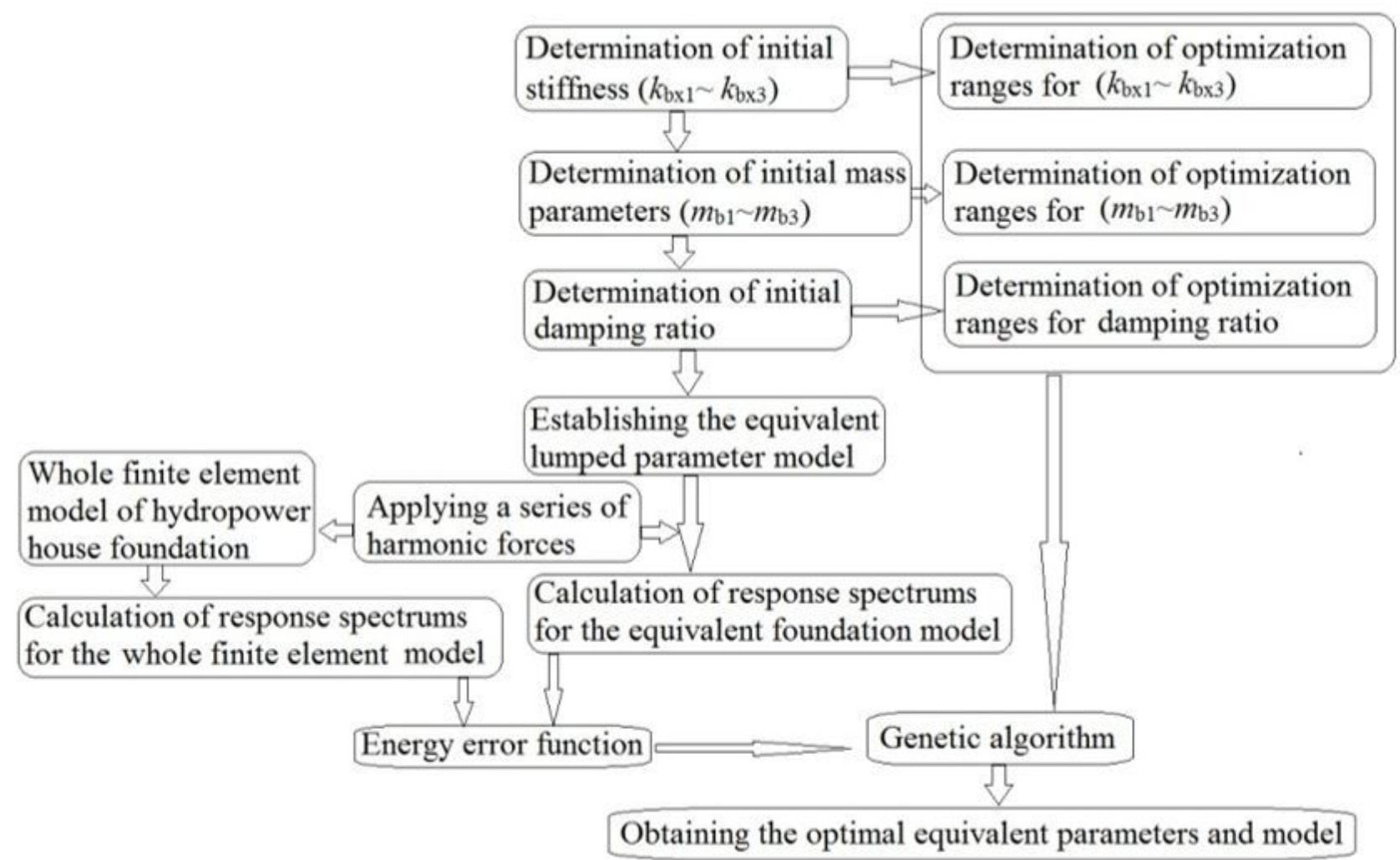

Figure 2

Procedure for establishing an optimal energy-equivalent model for foundation subsystem 


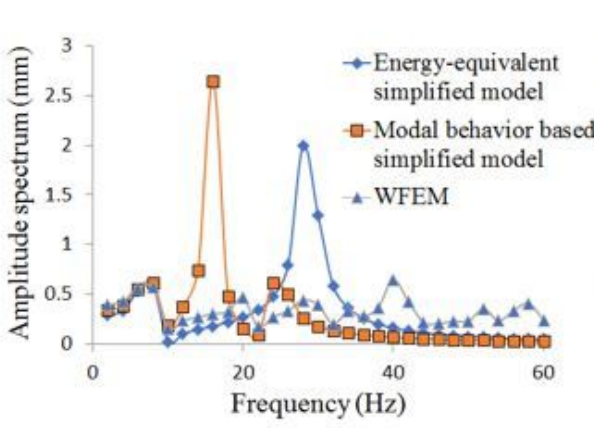

(a) 1-1

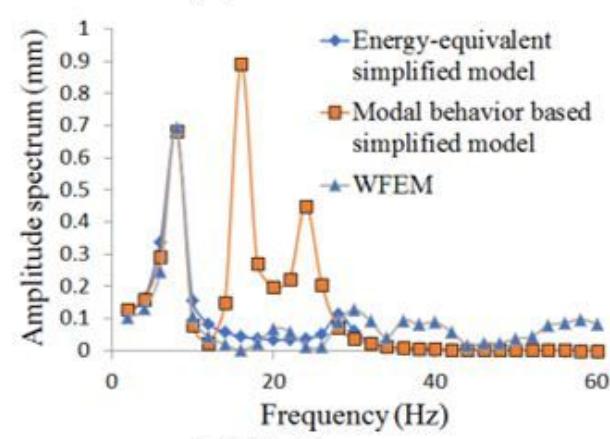

(d) 2-1

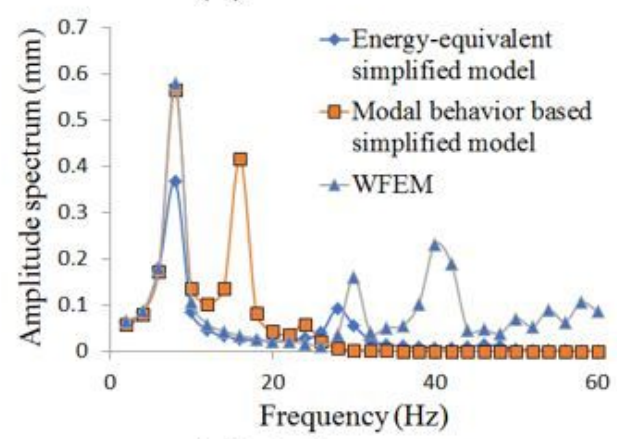

(g) 3-1

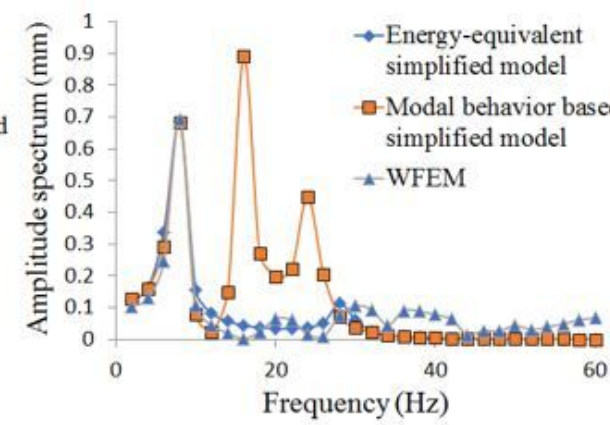

(b) $1-2$

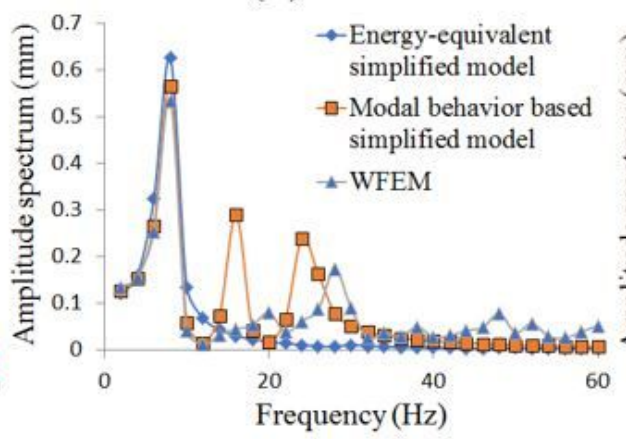

(e) 2-2

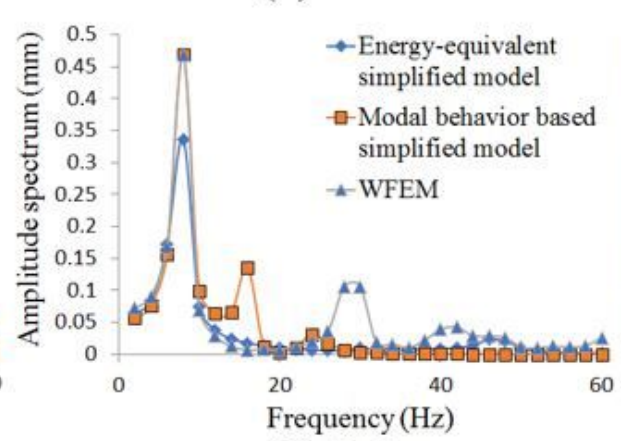

(h) 3-2

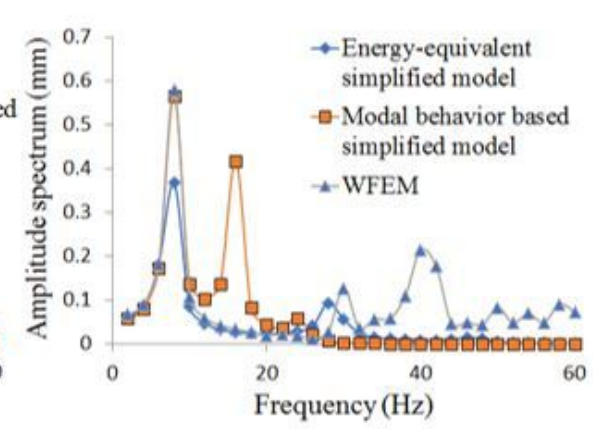

(c) 1-3

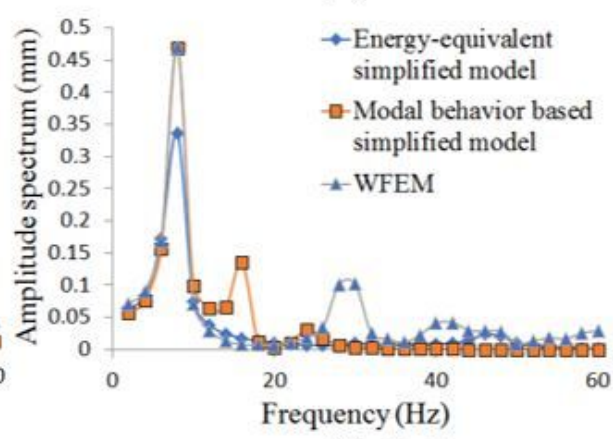

(f) 2-3

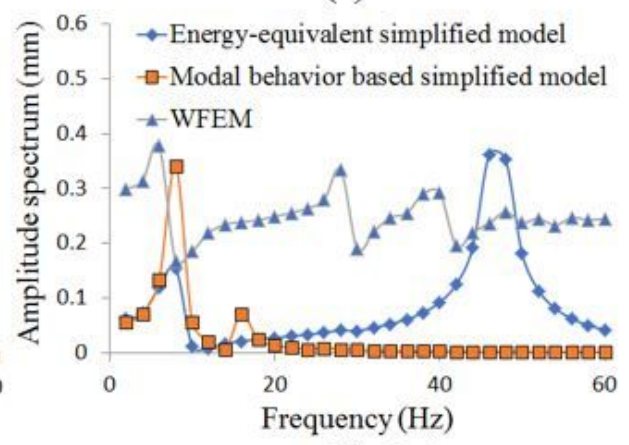

(i) 3-3

\section{Figure 3}

Model comparison for foundation subsystem in frequency-domain (In the subtitle " $1,2,3$ " denote upper guide bearing foundation, lower guide bearing foundation and turbine guide bearing foundation, respectively. The symbol "1-2" means "the dynamic responses of the lower guide bearing foundation when upper guide bearing foundation is subjected to harmonic loadings.") 


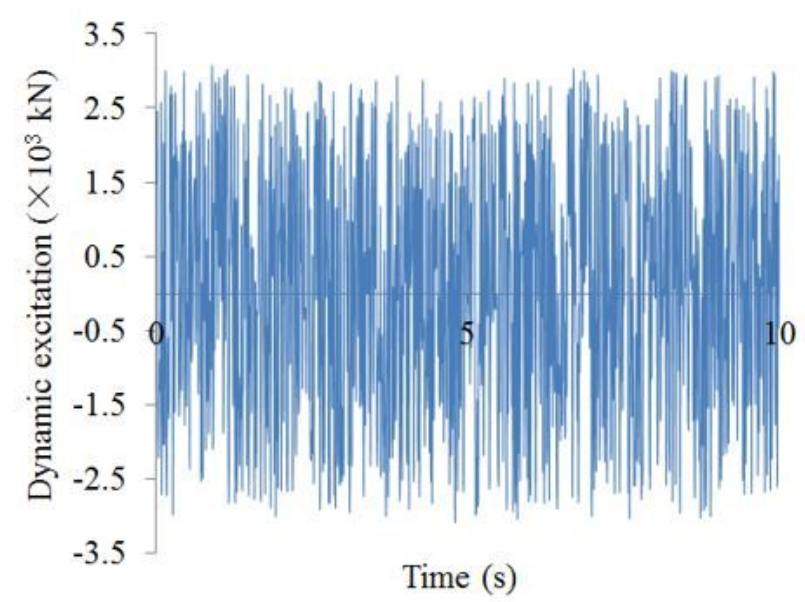

(a)

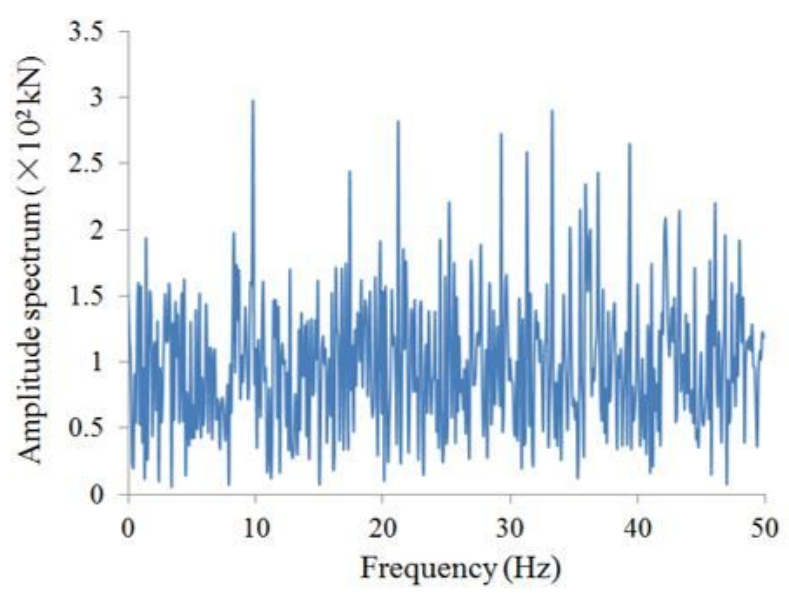

(b)

\section{Figure 4}

The first time-varying excitation constructed by white noise. (a) time-history curve of this excitation; (b) spectrum characteristic of this excitation
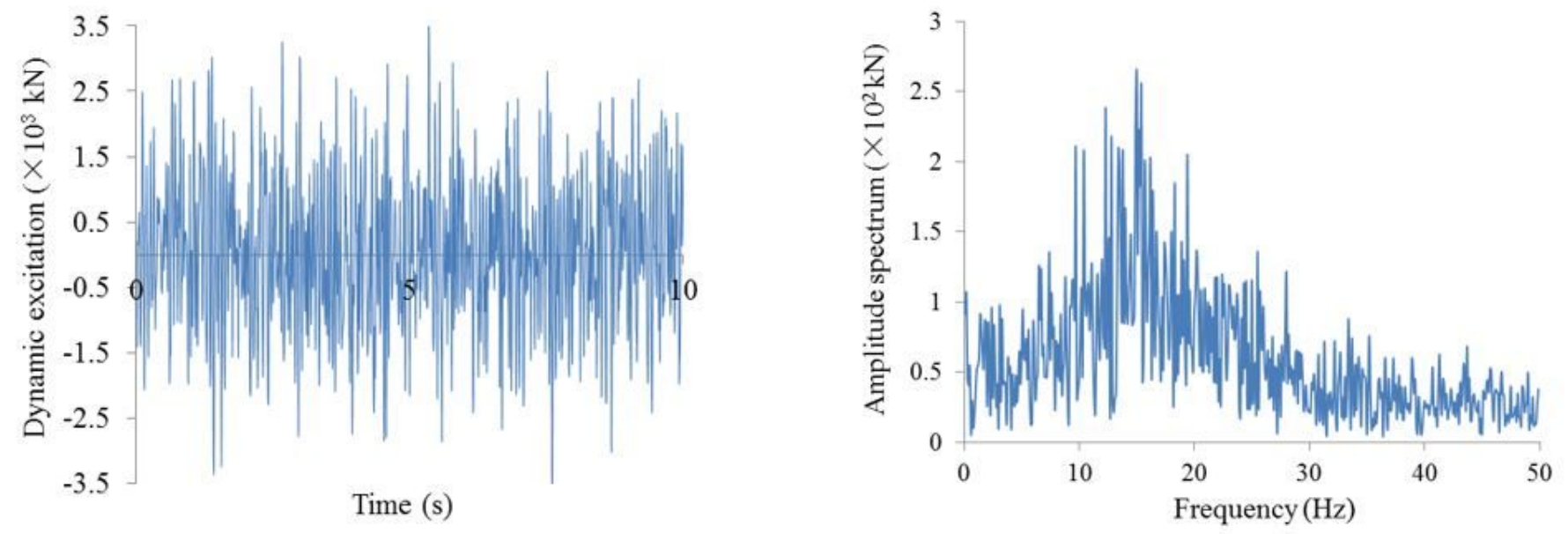

Figure 5

The second time-varying excitation constructed by bounded noise. (a) time-history curve of this excitation; (b) spectrum characteristic of this excitation 


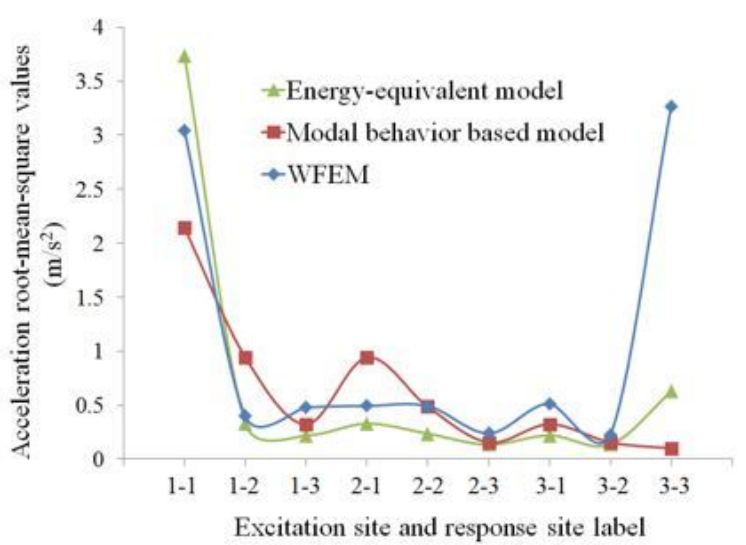

(a) Response to the first random excitation;

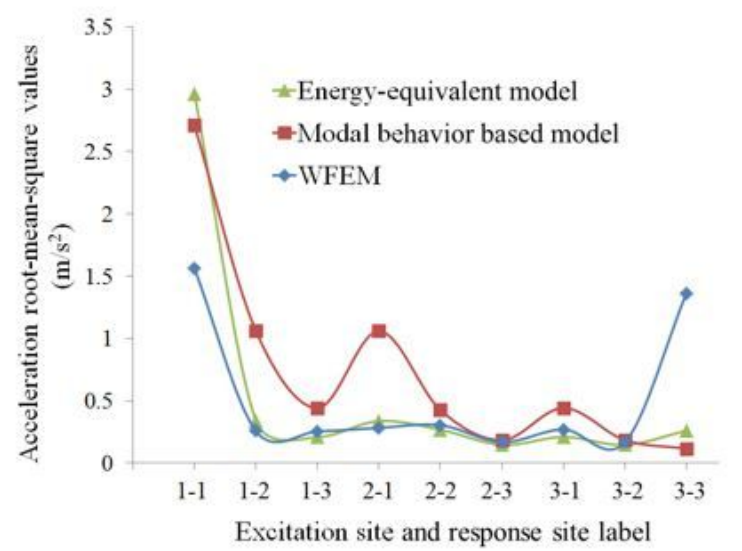

(b) Response to the second random excitation

Figure 6

Acceleration root-mean-square values of time-history responses for different models (See Fig. 3 for description of the $\mathrm{X}$-axis markers)

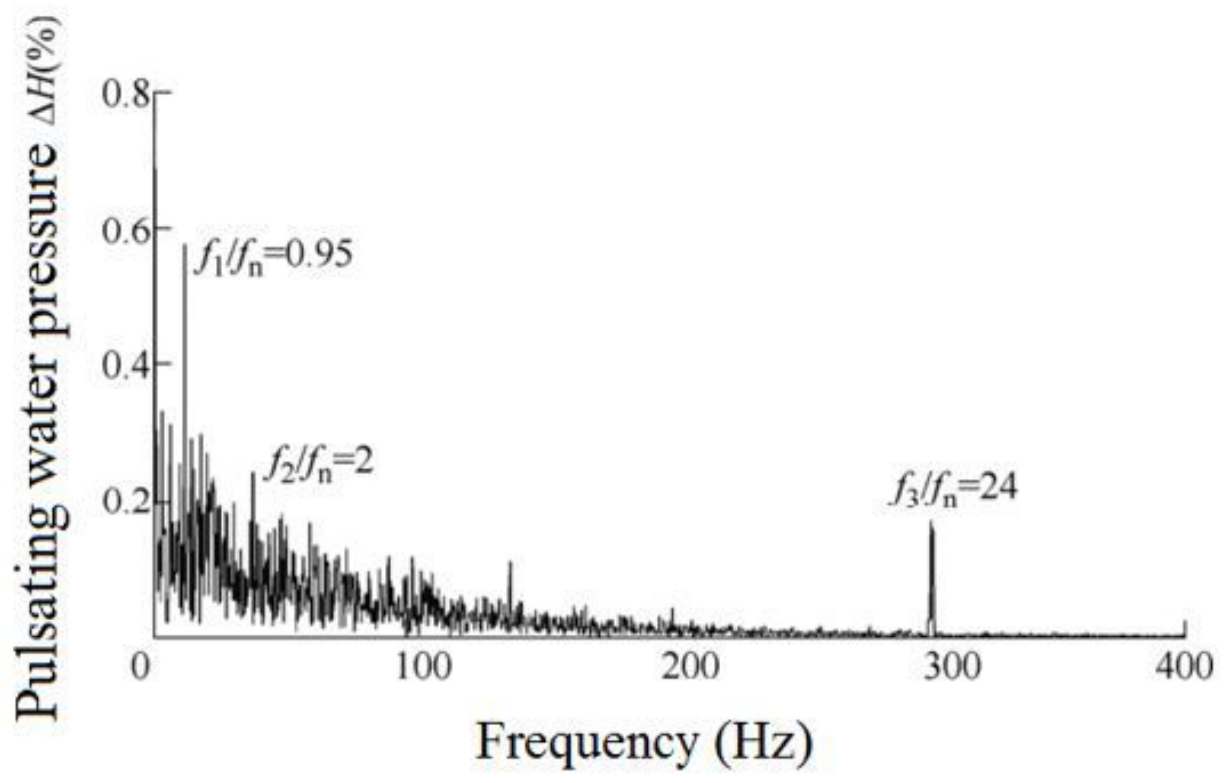

Figure 7

Typical pressure fluctuation of the turbine runner tested on prototype machine [22] 


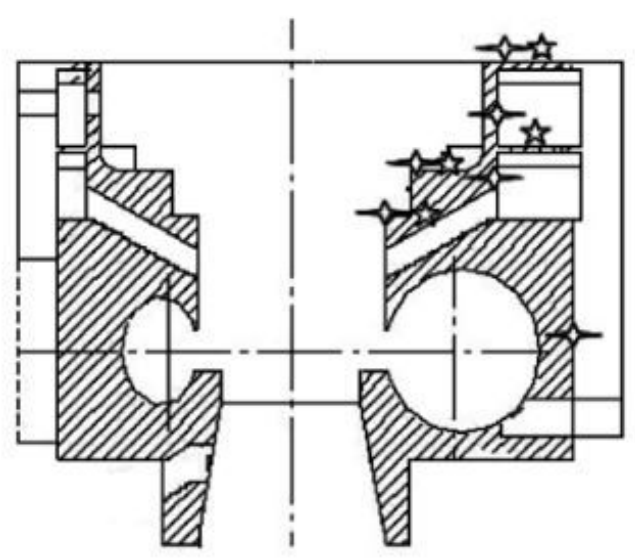

(a) Foundation subsystem

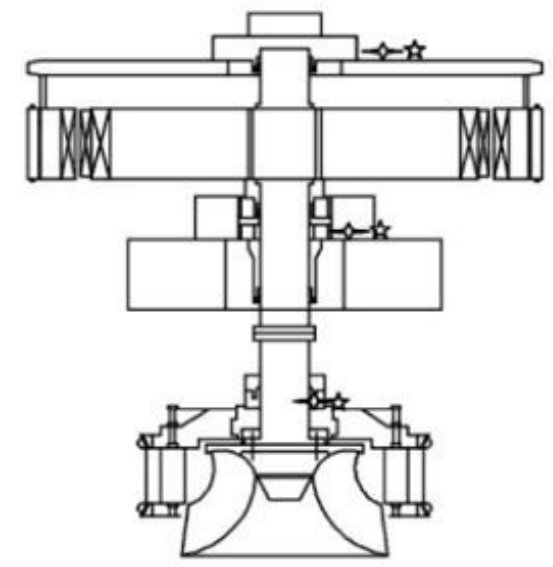

(b) Shaft subsystem

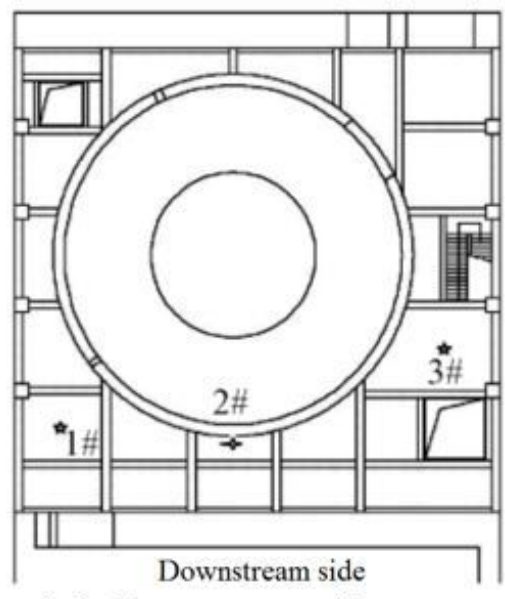

(c) Generator floor

Figure 8

Sketch of testing point arrangement [26]

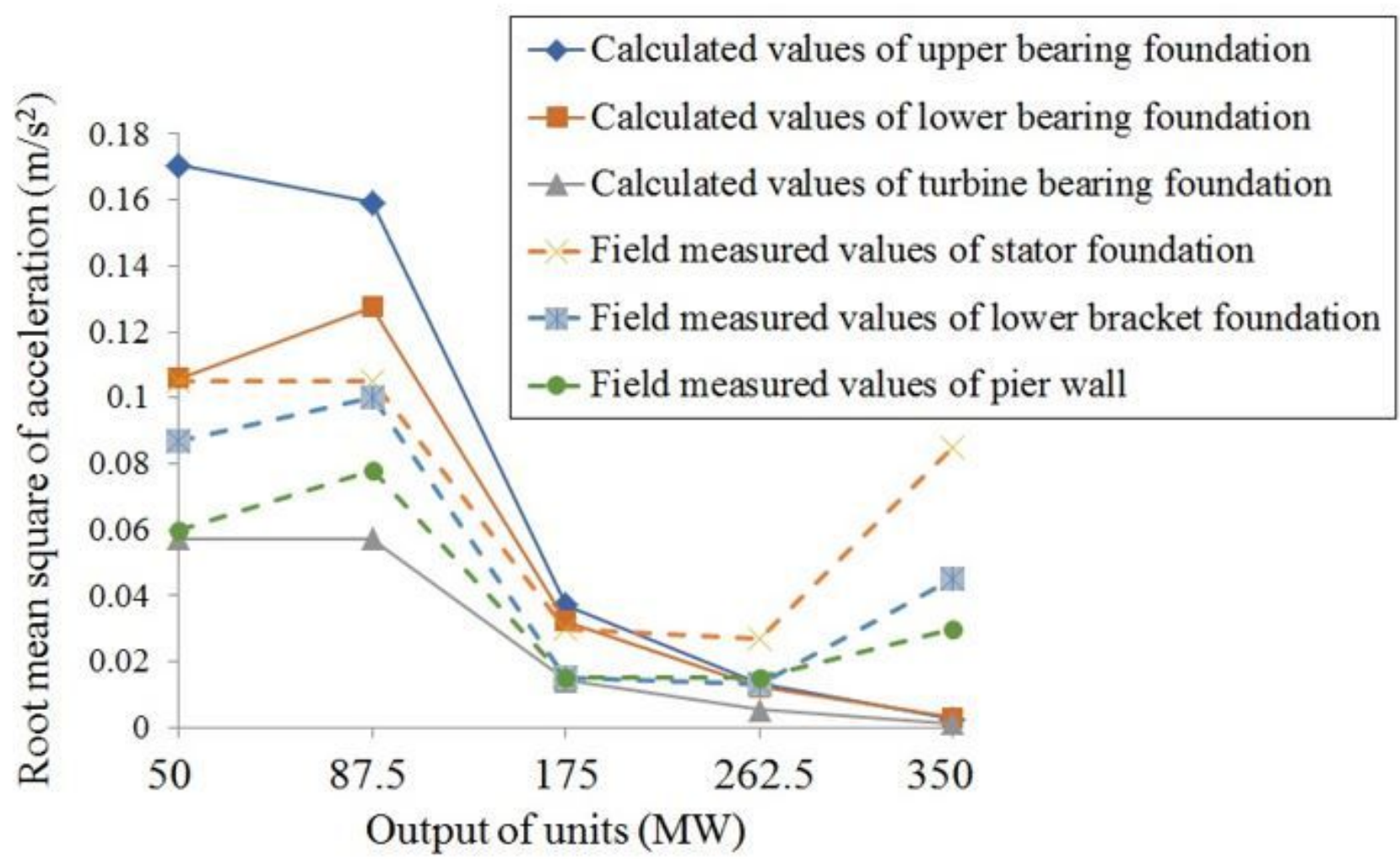

Figure 9

Comparison of acceleration root mean square of foundation longitude-river vibration calculated by proposed CSFS model with field tested results in Song et al. [26]. 


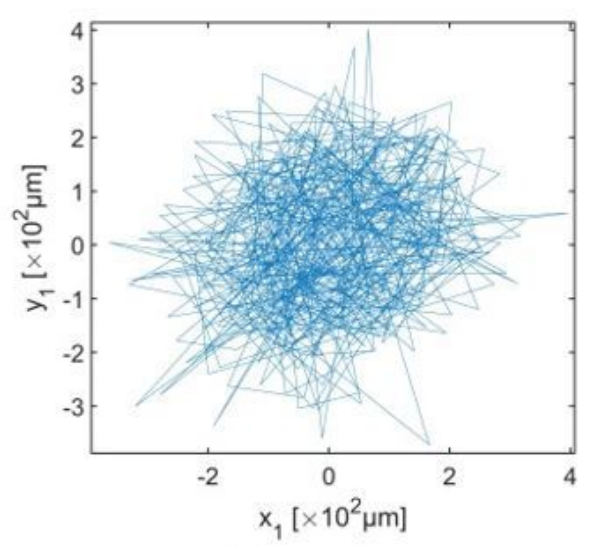

(a)

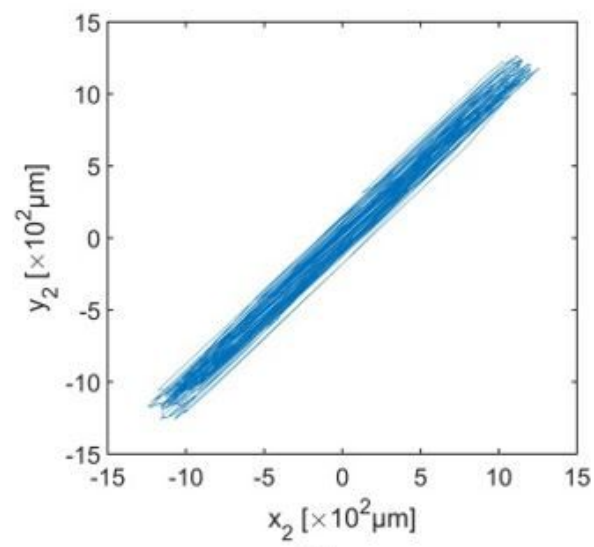

(b)

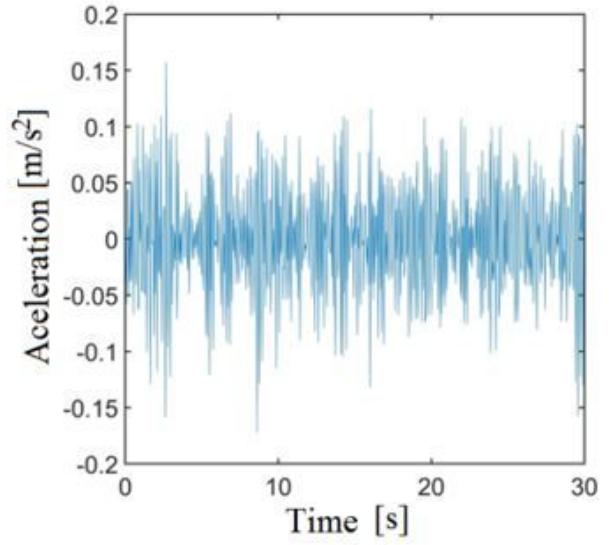

(c)

Figure 10

Calculated vibration results of the CSFS in the case of $87.5 \mathrm{MW}$ load. (a) Phrase diagram of the axis coordinates for generator rotor; (b) Phrase diagram of the axis coordinates for turbine runner; (c) Aceleration-time history of lower bearing foundation.

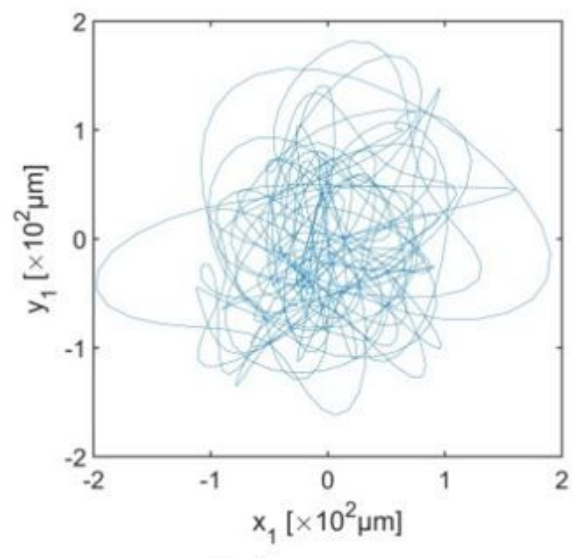

(a)

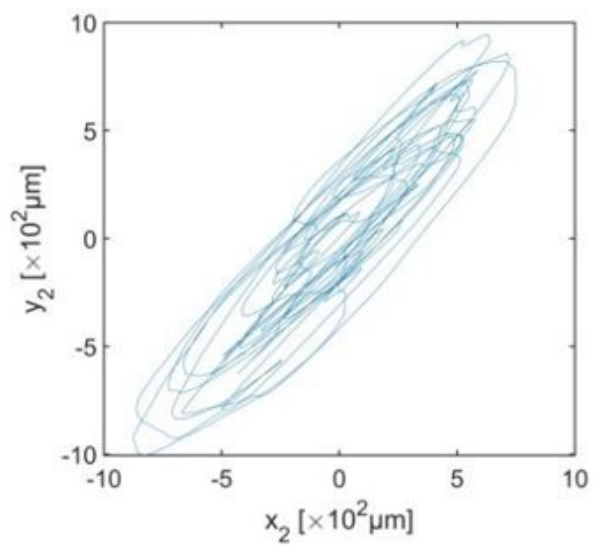

(b)

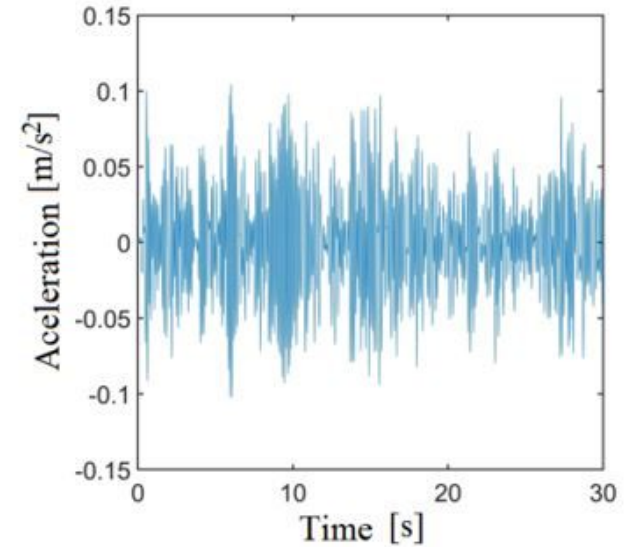

(c)

\section{Figure 11}

Calculated vibration results of the CSFS in the case of 350MW load. (a) Phrase diagram of the axis coordinates for generator rotor; (b) Phrase diagram of the axis coordinates for turbine runner; (c) Aceleration-time history of lower bearing foundation. 


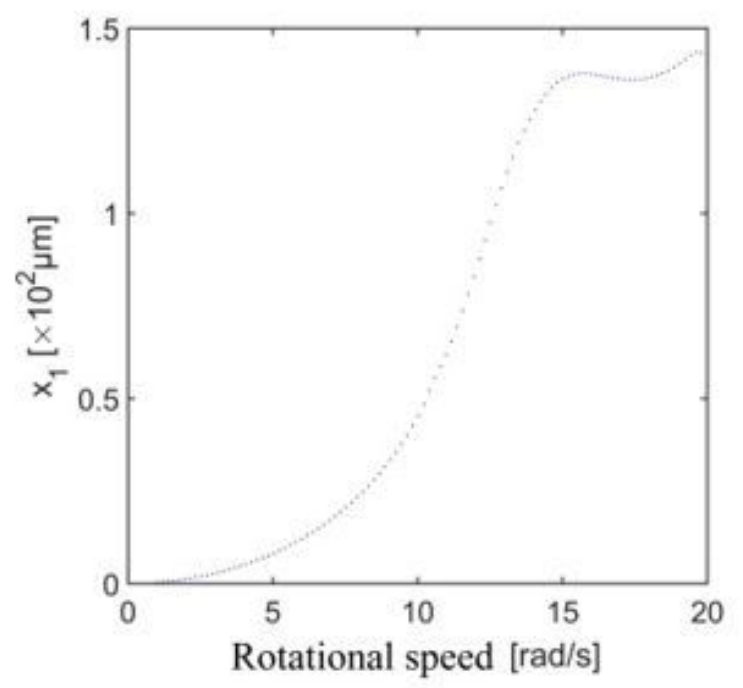

(a) Coupled shaft-foundation system

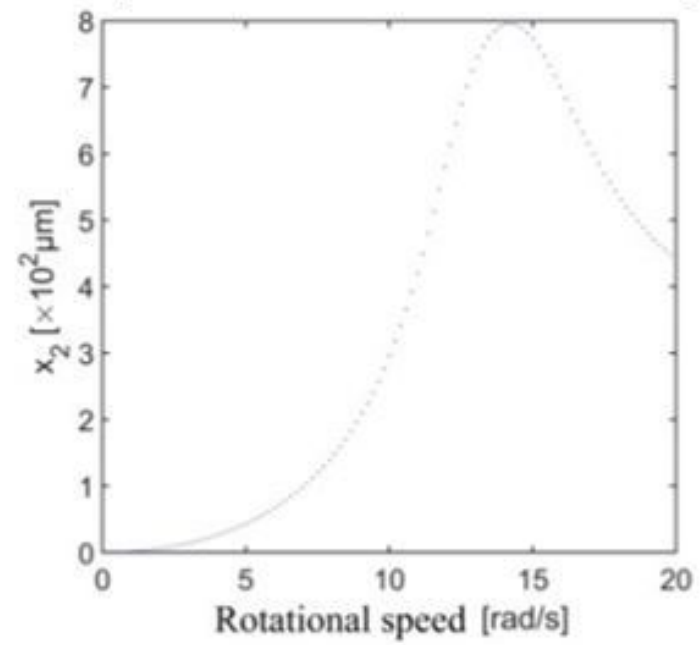

(a) Coupled shaft-foundation system

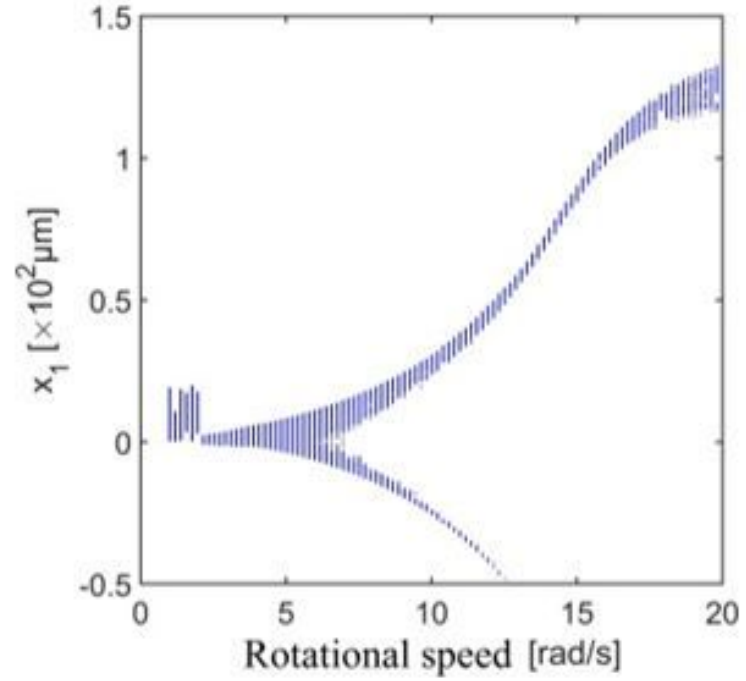

(b) Individual shaft system

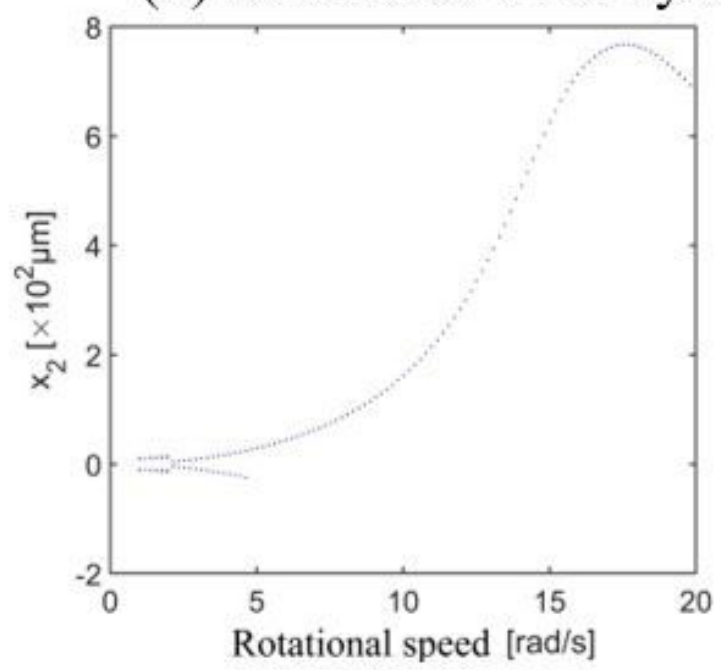

(b) Individual shaft system

\section{Figure 12}

12(a) Bifurcation diagrams of generator rotor axis coordinate at different rotational speeds 12(b) Bifurcation diagrams of turbine runner axis coordinate at different rotational speeds 


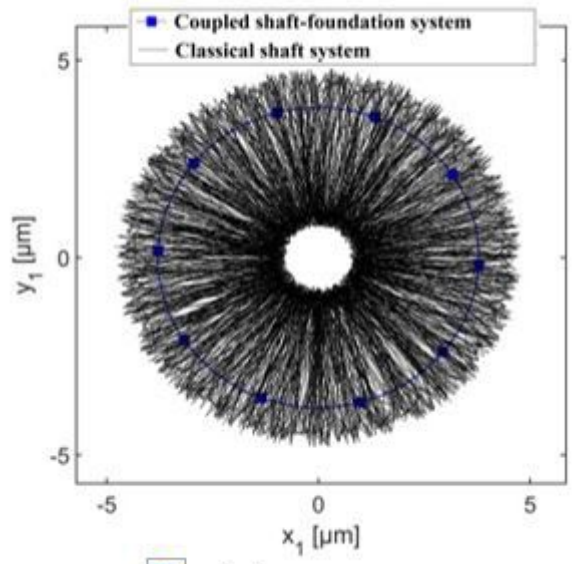

(a) $\omega=3.5 \mathrm{rad} / \mathrm{s}$

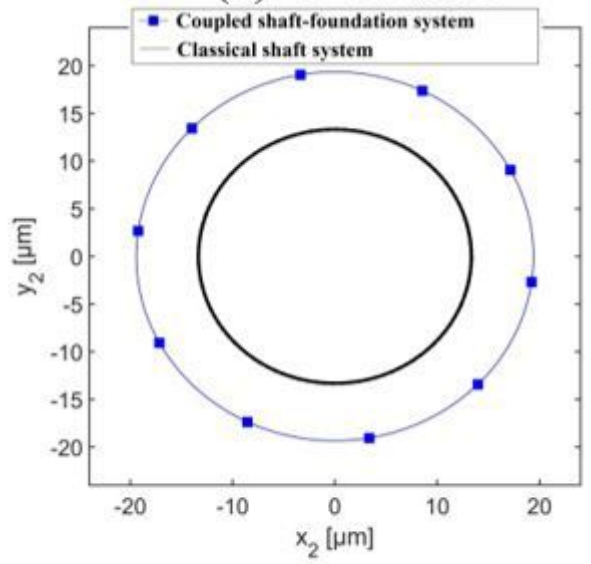

(a)

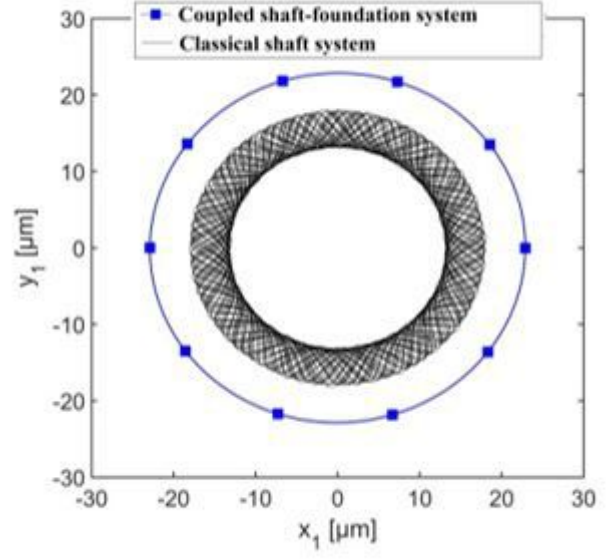

(b) $\omega=7.85 \mathrm{rad} / \mathrm{s}$

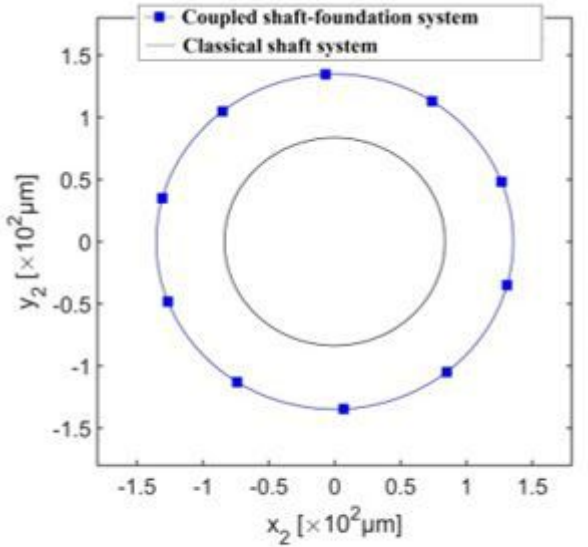

(b) $\omega=7.85 \mathrm{rad} / \mathrm{s}$

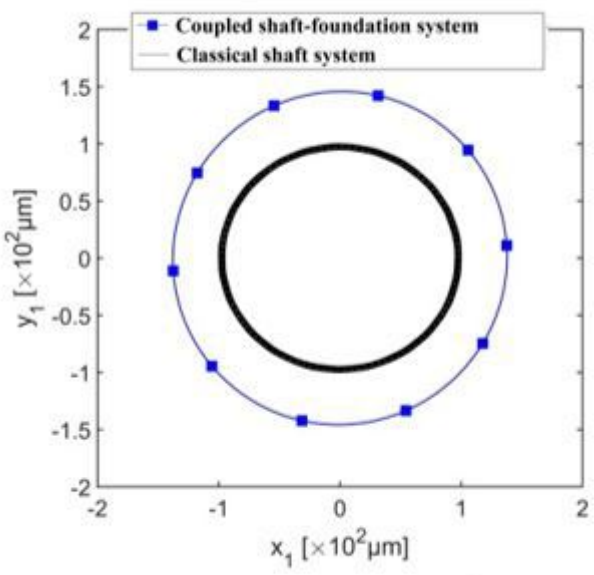

(c) $\omega=15.7 \mathrm{rad} / \mathrm{s}$

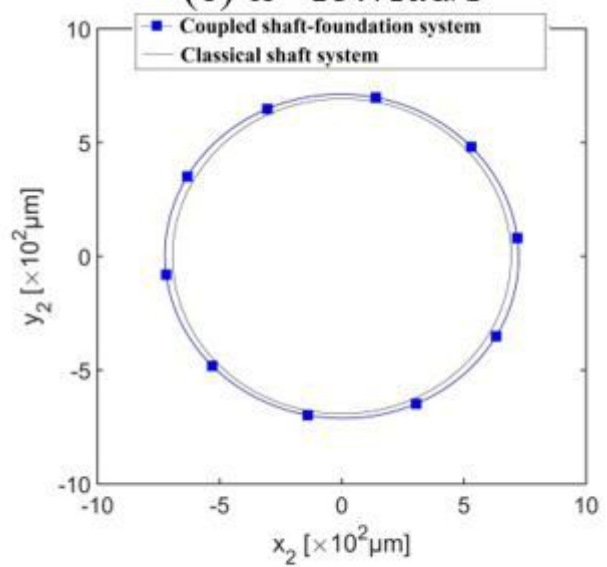

(c) $\omega=15.7 \mathrm{rad} / \mathrm{s}$

\section{Figure 13}

13(a) Phrase diagrams of the axis coordinates for generator rotor at different rotational speeds 13(b) Phrase diagrams of the axis coordinates for turbine runner at different rotational speeds 

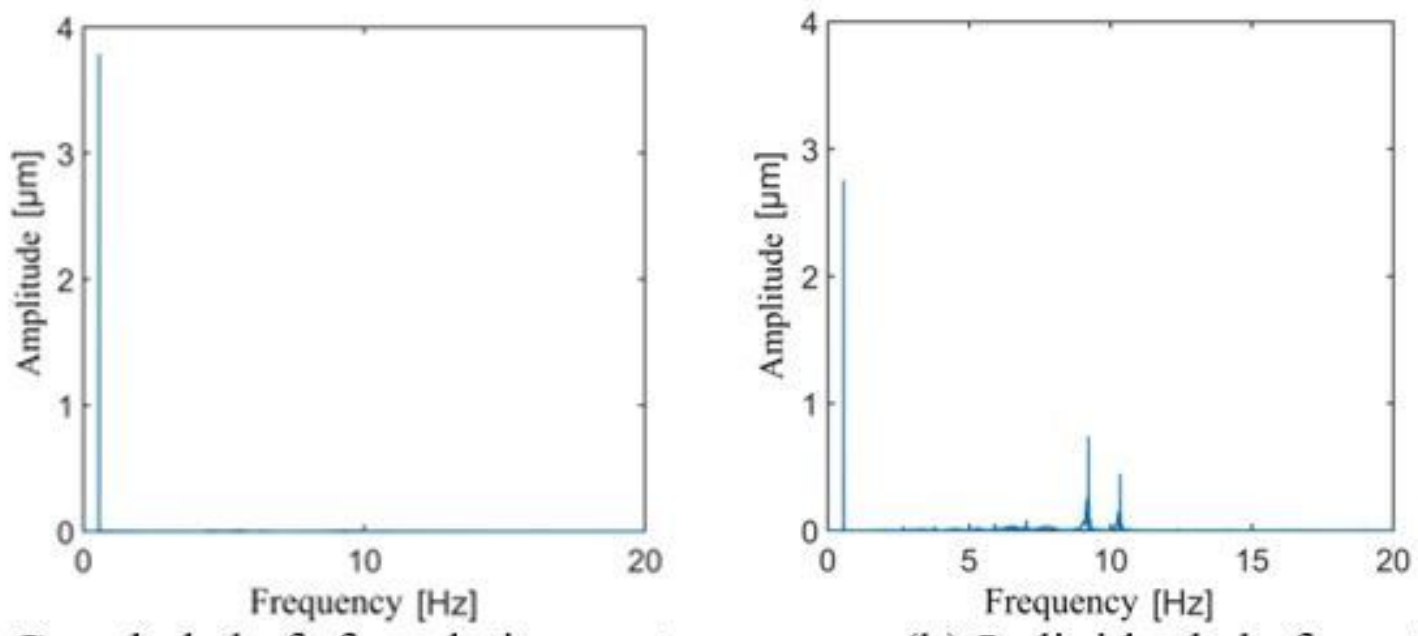

(a) Coupled shaft-foundation system

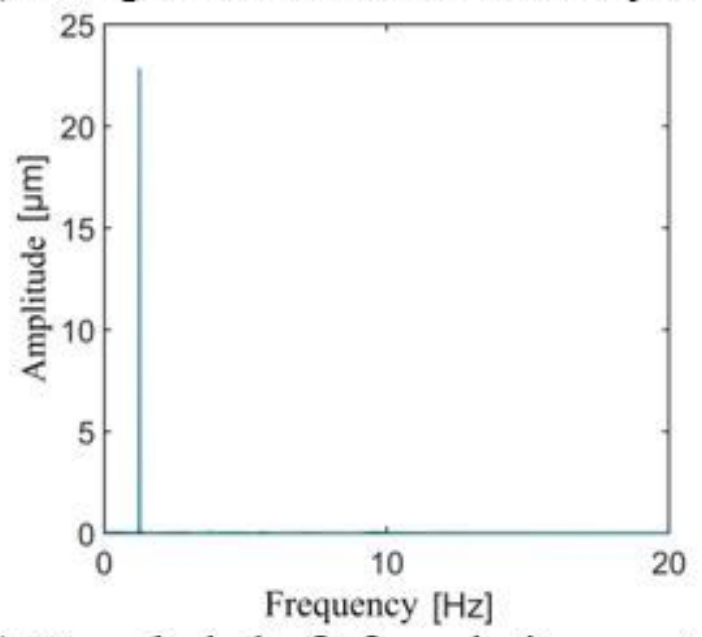

(b) Individual shaft system

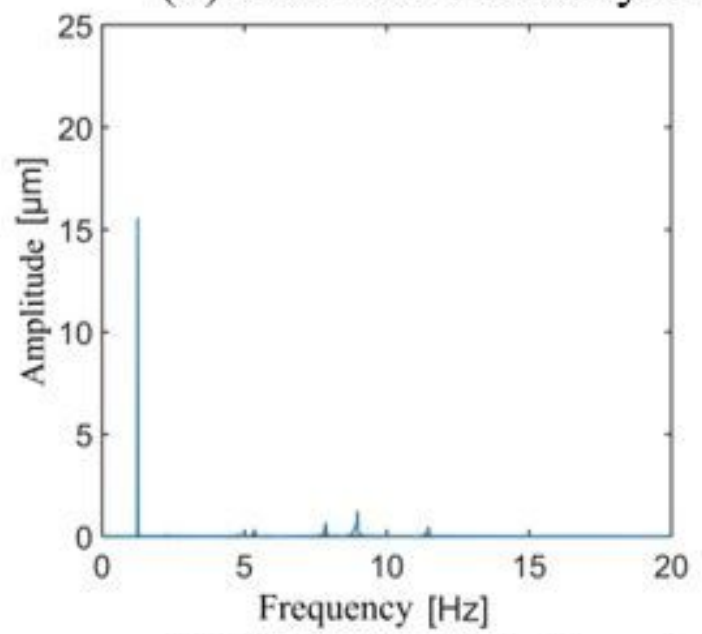

(a) Coupled shaft-foundation system

(b) Individual shaft system

\section{Figure 14}

14(a) Amplitude-frequency figures of generator rotor lateral vibration ( $\omega=3.5 \mathrm{rad} / \mathrm{s}) 14(\mathrm{~b})$ Amplitudefrequency figures of generator rotor lateral vibration $(\omega=7.85 \mathrm{rad} / \mathrm{s})$ 